\title{
Flavor nonsinglet parton distribution functions from lattice QCD at physical quark masses via the pseudodistribution approach
}

\author{
Manjunath Bhat $\odot,{ }^{1}$ Krzysztof Cichy $\odot,{ }^{1}$ Martha Constantinou $\odot,{ }^{2}$ and Aurora Scapellato ${ }^{1}$ \\ ${ }^{1}$ Faculty of Physics, Adam Mickiewicz University, ulica Uniwersytetu Poznańskiego 2, \\ 61-614 Poznań, Poland \\ ${ }^{2}$ Temple University, 1925 North 12th Street, Philadelphia, Pennsylvania 19122-1801, USA
}

(Received 12 May 2020; accepted 4 February 2021; published 24 February 2021)

\begin{abstract}
One of the great challenges of QCD is to determine the partonic structure of the nucleon from first principles. In this work, we provide such a determination of the flavor nonsinglet $(u-d)$ unpolarized parton distribution function (PDF), utilizing the nonperturbative formulation of QCD on the lattice. We apply Radyushkin's pseudodistribution approach to lattice results obtained using simulations with the light quark mass fixed to its physical value; this is the first ever attempt for this approach directly at the physical point. The extracted coordinate-space matrix elements are used to find the relevant physical Ioffe time distributions from a matching procedure. The full Bjorken- $x$ dependence of PDFs is resolved using several reconstruction methods to tackle the ill-conditioned inverse problem encountered when using discrete lattice data. We consider both the valence distribution $q_{v}$ and the combination with antiquarks $q_{v}+2 \bar{q}$, related to, respectively, the real and imaginary part of extracted matrix elements. Good agreement is found with PDFs from global fits already within statistical uncertainties and it is further improved by quantifying several systematic effects. The results presented here are the first ever $a b$ initio determinations of PDFs fully consistent with global fits in the whole $x$ range. Thus, they pave the way to investigating a wider class of partonic distributions, such as e.g., singlet PDFs and generalized parton distributions. Therefore, essential and yet missing first-principle insights can be achieved, complementing the rich experimental programs dedicated to the structure of the nucleon.
\end{abstract}

DOI: 10.1103/PhysRevD.103.034510

\section{INTRODUCTION}

Despite the fact that the nucleon is the main building block of visible matter and is responsible for almost all of the mass of the visible Universe, it is only now that several aspects of its internal structure are beginning to be thoroughly explored. The wealth of data from presentday experiments, e.g., from the Large Hadron Collider and the Jefferson Laboratory 6 and $12 \mathrm{GeV}$ programs, allows us to unravel many details that so far eluded any insight. Moreover, the planned and recently approved Electron-Ion Collider at the Brookhaven National Laboratory, that will start operation in around ten years, is oriented at answering important questions about the nucleon structure, such as of the origin of the proton mass, the spin distribution and the role of gluons [1]. Along with the experimental efforts, there is constant progress in the theoretical understanding, based on exhaustive analyses of empirical data and various

Published by the American Physical Society under the terms of the Creative Commons Attribution 4.0 International license. Further distribution of this work must maintain attribution to the author(s) and the published article's title, journal citation, and DOI. Funded by SCOAP . approaches to describe the physics of the strong dynamics of partons (quarks and gluons), governed by the theory of quantum chromodynamics (QCD). One of the most important tools is perturbation theory, which is, however, limited to high-energy scales, at which the perturbative expansion can evince convergence. Meanwhile, it is clear that all energy scales contribute to the dynamics of the nucleon and hence the description of the nonperturbative aspects is of essential importance. This can take the form of phenomenological models, which have led to important successes. However, a truly $a b$ initio knowledge can still be, in principle, extracted directly from the QCD Lagrangian. The most successful nonperturbative approach to QCD is to formulate it on a discrete spacetime grid, the lattice. This leads to a regularization of the QCD path integral and results in multidimensional integrals that can be evaluated numerically, usually with Monte Carlo simulations. Such computations, however, are necessarily done in Euclidean spacetime, which poses a fundamental problem for partonic physics, most naturally formulated in terms of light-cone correlations. The latter cannot be accessed in Euclidean lattice QCD (LQCD) and, thus, the information on the nucleon structure from LQCD has been limited for many years. Most of the insights were obtained from lattice 
calculations of moments of parton distribution functions (PDFs) and generalized parton distributions. In principle, the full distributions can be reconstructed from a sufficiently large number of their moments, as obtained from LQCD. However, in practice, the computations are limited to only the lowest 2-3 moments. For higher moments, the breaking of rotational symmetry implied by the lattice leads to unavoidable power-divergent mixings with lower-dimensional operators and, what is more, the signal-to-noise ratio for such higher moments is quickly decaying. For recent calculations of the low moments by the Extended Twisted Mass Collaboration (ETMC), we refer the interested reader to Refs. [2-7].

Extending the calculations of moments to the full $x$ dependence of the partonic distributions has been a subject of intense studies over the years. Even though the early proposals date back to the previous century, the recent revival of this topic, one that brought considerable progress to this field, came with the proposal of Ji [8] to calculate socalled quasidistributions. They are related to the desired light-cone distributions but computable on a Euclidean lattice. The underlying idea is to replace the light-cone correlations in the definition of a distribution by spatial ones between boosted nucleon states. One can then exploit the fact that the obtained quasidistributions share the infrared physics with their light-cone counterparts. As a consequence, their difference is in the ultraviolet and can be matched using perturbation theory, utilizing the so-called large momentum effective theory (LaMET) [9]. Ji's proposal sparked a huge tide of theoretical and numerical efforts to understand crucial aspects of this approach, such as renormalizability and appropriate renormalization prescriptions, matching, nucleon mass corrections, highertwist effects, and finite volume effects, as well as to extract the distributions in various setups; see e.g., Refs. [10-70].

Quasidistributions can be thought of as generalization of the light-cone ones to the finite-momentum frame. As shown by Radyushkin in a series of papers [36,71-77], the same matrix elements that specify a quasidistribution can also be used to define another generalization of its lightcone counterpart, the so-called pseudodistribution. These matrix elements can be viewed as functions of two Lorentz invariants. The first one is the spacetime interval $z^{2}$, where $z^{\mu}$ can be taken as $\left(0,0,0, z_{3}\right)$ and describes the separation between the quarks in the inserted operator (containing the Wilson line to guarantee gauge invariance). The other Lorentz invariant is the product $\nu \equiv-p \cdot z$ (with $p^{\mu}$ being the nucleon boost) and the variable $\nu$ is called the Ioffe time [78]. The matrix elements written as the function of $z^{2}$ and $\nu$ are called Ioffe-time distributions (ITDs).

The underlying difference between quasi- and pseudodistributions is that the former are defined as the Fourier transform of the matrix elements in $z_{3}$, while to obtain the latter, one takes the transform in the Ioffe time. This has far-reaching consequences and makes the approaches inequivalent, even though they can be computed from the same matrix elements. In particular, pseudodistributions have the canonical support in the Bjorken- $x$ fraction, $-1 \leq x \leq 1$, as opposed to quasidistributions that can be nonzero also for $x$ outside of this range. The matching of pseudodistributions to the light-cone frame $[31,36,75,79]$ is performed at the level of ITDs. As we shall see below, the matching is numerically a smaller effect than for quasiPDFs. In particular, it depends less significantly on the region of $x$. It is also an important difference that pseudodistributions can fully utilize lattice data at all nucleon boosts; i.e., they contain physical information also from low momenta, effectively at small Ioffe times. Thus, the matching of ITDs is not based on LaMET but still on a factorization of the finite-momentum distribution into a light-cone one and a perturbatively computable matching coefficient. The pseudodistribution approach to extract the valence unpolarized PDF of the nucleon was explored first in the quenched setup [80] and recently it was extended to include the effects of dynamical quarks at nonphysical masses [81] and to investigate the PDFs of the pion [82]. Even more recently, it was also applied in a setup with light quark mass close to, but not yet directly at its physical value, with the corresponding pion mass of $170 \mathrm{MeV}$ [83]. In addition, moments of ITDs were computed [37] and the general issue of reconstructing distributions from ITDs under incomplete Fourier transforms was analyzed in related studies by the same group [44] and, most recently, the pseudo-PDF data were used to reconstruct PDFs using neural networks [84].

Apart from approaches based on ITDs, we also mention other proposed methods that can lead to determinations of the full $x$ dependence of partonic distributions. Instead of using a Wilson line to ensure gauge invariance, they can employ auxiliary propagators, of fictitious scalar [85], heavy [86] or light quarks [87-89]. Approaches based on the hadronic tensor also exist $[90,91]$. Similarly to quasiand pseudodistributions, these methods are also intensely investigated by various groups; see e.g., Refs. [92-97]. An extensive review of these efforts, with emphasis on the most investigated approach of quasidistributions, can be found in Refs. [98,99]. Recently, a review devoted to the principles and various applications of LaMET also appeared [100].

In this paper, we apply the pseudodistribution approach for the first time to lattice data obtained with light quark mass fixed to its physical value. We always consider the flavor nonsinglet combination $u-d$. We provide an extraction of two kinds of nonsinglet distributions - the valence one, denoted here by $q_{v}(x)$, and its combination with antiquarks $q_{v}(x)+2 \bar{q}(x)$. Taking their linear combinations, we also show results for the full distribution, $q(x)=q_{v}(x)+\bar{q}(x)$, and for the sea quark PDF, $q_{s}(x)=\bar{q}(x)$. We explore several systematic effects inherited in lattice computations and we address the issue of reconstructing the PDFs from ITDs, subject to an 
ill-defined inverse problem. We show that this leads ultimately to full consistency between all considered distributions and the corresponding ones from global fits, in the whole range of Bjorken- $x$. Even though at the present stage full quantification of all systematics is not yet possible, the results obtained in this work are unambiguously optimistic and demonstrate the potential of the techniques for an issue that was for many years thought to be too difficult for lattice QCD calculations.

The outline of the remainder of the paper is the following. In Sec. II, we discuss theoretical principles of pseudo-PDFs and practical aspects of their computations. Then, we present the lattice details of the calculation in Sec. III. In Sec. IV, we show our results for the pseudodistributions and their matching to light-cone ITDs and we compare different methods for the reconstruction of PDFs. We also discuss systematic effects from the choice of the Ioffe time range, the value of the strong coupling constant and from other sources and in Sec. IV D, we present our final PDFs. We also show results for the low moments obtained from polynomial fits to fixed $-z^{2}$ ITDs and from integrating the final reconstructed distributions. Finally, we conclude and discuss future prospects in Sec. V.

\section{THEORETICAL SETUP AND ANALYSIS TECHNIQUES}

We start by summarizing the relevant steps in the procedure leading from lattice-extracted matrix elements (pseudo-ITDs) to the light-cone distributions, ITDs and PDFs after a suitable reconstruction. We refer the reader to the review of Ref. [76] for an extensive discussion on the theoretical principles and properties of pseudodistributions.

The underlying matrix elements computed on the lattice are defined (in Euclidean spacetime) as

$$
\mathcal{M}\left(\nu, z^{2}\right)=\left\langle P\left|\bar{\psi}(0, z) \gamma_{0} W(z, 0) \psi(0,0)\right| P\right\rangle,
$$

where $|P\rangle$ is a boosted nucleon state with four-momentum $P_{\mu}=\left(P_{0}, 0,0, P_{3}\right)$ and $W(z, 0)$ is a straight Wilson line. The Wilson line is chosen along the direction of the boost and has length $z$ [i.e., we take $z^{\mu}=(0,0,0, z)$ and $z$ will henceforth refer to the length of $z^{\mu}$. With this choice of kinematics, the Ioffe time $\nu=P_{3} z$. The Dirac structure $\gamma_{0}$ leads to a faster convergence to the light-cone ITD, as compared to another plausible choice of the $\gamma_{3}$ structure [71]. It was also found that $\gamma_{0}$ avoids a finite mixing with the twist-3 scalar operator due to the breaking of chiral symmetry by some fermionic discretizations [23].

The matrix element defined by Eq. (1) exhibits two kinds of divergences: the standard logarithmic one and a power divergence related to the Wilson line. However, it has been shown to be multiplicatively renormalizable to all orders in perturbation theory [21,22]. In Ref. [80], it was suggested that the divergences can be canceled by forming a double ratio with zero-momentum and local $(z=0)$ matrix elements:

$$
\mathfrak{M}\left(\nu, z^{2}\right)=\frac{\mathcal{M}\left(\nu, z^{2}\right) / \mathcal{M}(\nu, 0)}{\mathcal{M}\left(0, z^{2}\right) / \mathcal{M}(0,0)} .
$$

We follow this renormalization procedure and we refer to $\mathfrak{M}\left(\nu, z^{2}\right)$ as reduced matrix elements or pseudo-ITDs. It is expected, although not proven, that the double ratio not only removes the divergences, but also part of the higher-twist contamination, which is generically of $\mathcal{O}\left(z^{2} \Lambda_{\mathrm{QCD}}^{2}\right)$ [80]. The double ratio defines a renormalization scheme where the renormalization scale is proportional to the inverse length of the Wilson line.

To get from pseudo-ITDs to light-cone ITDs and finally to light-cone PDFs, a matching procedure is required, similarly to the quasi-PDFs case. The reduced matrix elements, defined at different scales $1 / z$, need to be evolved to a common scale, $1 / z^{\prime}$, and it is desirable to also convert them to the renormalization scheme commonly used for PDFs, the $\overline{\mathrm{MS}}$ scheme, where its renormalization scale will be denoted by $\mu$. The full matching equation, to one-loop order in perturbation theory, reads $[31,36,75,79]$

$$
\begin{aligned}
\mathfrak{M}\left(\nu, z^{2}\right)= & Q\left(\nu, \mu^{2}\right)+\frac{\alpha_{s} C_{F}}{2 \pi} \int_{0}^{1} d u \\
& \times\left[\ln \left(z^{2} \mu^{2} \frac{e^{2 \gamma_{E}+1}}{4}\right) B(u)+L(u)\right] Q\left(u \nu, \mu^{2}\right),
\end{aligned}
$$

where $Q\left(\nu, \mu^{2}\right)$ is the $\overline{\mathrm{MS}}$-scheme light-cone ITD and the functions convoluted with $Q$ are

$$
\begin{gathered}
B(u)=\left[\frac{1+u^{2}}{1-u}\right]_{+} \\
L(u)=\left[4 \frac{\ln (1-u)}{1-u}-2(1-u)\right]_{+}
\end{gathered}
$$

with the plus prescription defined as

$$
\int_{0}^{1}[f(u)]_{+} Q(u \nu)=\int_{0}^{1} f(u)(Q(u \nu)-Q(\nu)) .
$$

The matching equation consists of two parts. The part containing the kernel $B(u)$ evolves the pseudo-ITDs to a common scale $\mu$ and the part with $L(u)$ converts to the $\overline{\mathrm{MS}}$ scheme.

We invert the matching equation, and to look separately into the effect of evolution and scheme conversion, we introduce intermediate evolved ITDs, $\mathfrak{M}^{\prime}\left(\nu, z^{2}, \mu^{2}\right)$. Thus, 


$$
\begin{aligned}
\mathfrak{M}^{\prime}\left(\nu, z^{2}, \mu^{2}\right)= & \mathfrak{M}\left(\nu, z^{2}\right)-\frac{\alpha_{s} C_{F}}{2 \pi} \int_{0}^{1} d u \\
& \times \ln \left(z^{2} \mu^{2} \frac{e^{2 \gamma_{E}+1}}{4}\right) B(u) \mathfrak{M}\left(u \nu, z^{2}\right) .
\end{aligned}
$$

The evolved ITD has three arguments, the Ioffe time $\nu$, the common scale $\mu$ and the initial scale $z$. In principle, its value should be independent of the initial scale and we will test this conjecture up to our statistical precision. The scheme conversion then follows:

$$
Q\left(\nu, z^{2}, \mu^{2}\right)=\mathfrak{M}^{\prime}\left(\nu, z^{2}, \mu^{2}\right)-\frac{\alpha_{s} C_{F}}{2 \pi} \int_{0}^{1} d u L(u) \mathfrak{M}\left(u \nu, z^{2}\right),
$$

where again we will test the independence on the initial scale. For the reconstruction of the final PDF, discussed below, we will average the matched ITDs $Q\left(\nu, z^{2}, \mu^{2}\right)$ for cases where a given Ioffe time is achieved by different combinations of $\left(P_{3}, z\right)$ and denote such an average by $Q\left(\nu, \mu^{2}\right)$.

The matching equations discussed above involve a convolution of a kernel function with reduced ITDs; i.e., one needs to access the latter for all continuous values of the Ioffe time from 0 to $\nu$. We adopt two alternative approaches for the required interpolation at fixed $z^{2}$. We either do a linear interpolation between the ITDs at neighboring boosts or we perform fits to the $\nu$ dependence that utilizes all boosts. The real part is then fitted to even powers of the Ioffe time:

$$
\operatorname{Re} \mathfrak{M}\left(\nu, z^{2}\right)=1+c_{2} \nu^{2}+c_{4} \nu^{4}+\cdots,
$$

while the imaginary part to its odd powers:

$$
\operatorname{Im} \mathfrak{M}\left(\nu, z^{2}\right)=c_{1} \nu+c_{3} \nu^{3}+\cdots .
$$

We compare both approaches at the level of matrix elements and the final reconstructed partonic distributions and show that both of them lead to totally consistent outcomes. However, the fitting of the full boost dependence at fixed $z^{2}$ allows one to also extract PDF moments, as suggested in Ref. [37]. Moments of pseudo-PDFs are defined according to [37]

$$
\mathfrak{M}_{n}\left(z^{2}\right)=\left.(-i)^{n} \frac{\partial^{n} \mathfrak{M}\left(\nu, z^{2}\right)}{\partial \nu^{n}}\right|_{\nu=0}
$$

and are, thus, related to the fitting coefficients $c_{n}$. To extract moments of PDFs in the $\overline{\mathrm{MS}}$ scheme at a scale $\mu^{2}$, denoted here by $Q_{n}\left(\mu^{2}\right)$, one utilizes a matching equation $[37,83]$ that factorizes the pseudo-PDF moments into $\overline{\mathrm{MS}}$ moments and a perturbative coefficient expressing the Mellin moments of the ITD matching kernel:

$$
\mathfrak{M}_{n}\left(z^{2}\right)=C_{n}\left(\mu^{2} z^{2}\right) Q_{n}\left(\mu^{2}\right)
$$

up to $\mathcal{O}\left(z^{2} \Lambda_{\mathrm{QCD}}^{2}\right)$ higher-twist effects (HTE). At one-loop order,

$C_{n}\left(\mu^{2} z^{2}\right)=1-\frac{\alpha_{s} C_{F}}{2 \pi}\left[\gamma_{n} \ln \left(z^{2} \mu^{2} \frac{e^{2 \gamma_{E}+1}}{4}\right)+l_{n}\right]$,

with $\gamma_{n}$ being the moments of the kernel $B(u)$ of Eq. (4):

$$
\gamma_{n}=\frac{1}{(n+1)(n+2)}-\frac{1}{2}-2 \sum_{k=2}^{n+1} \frac{1}{k}
$$

and $l_{n}$ the moments of $L(u)$ of Eq. (5):

$l_{n}=2\left[\left(\sum_{k=1}^{n} \frac{1}{k}\right)^{2}+\sum_{k=1}^{n} \frac{1}{k^{2}}+\frac{1}{2}-\frac{1}{(n+1)(n+2)}\right]$.

We now move on to a discussion on how to extract partonic distributions from the evolved and schemeconverted ITDs. The ITDs $Q\left(\nu, \mu^{2}\right)$ are related to PDFs $q\left(x, \mu^{2}\right)$ by a Fourier transform in Ioffe time:

$$
Q\left(\nu, \mu^{2}\right)=\int_{-1}^{1} d x e^{i \nu x} q\left(x, \mu^{2}\right),
$$

where the antiquark distribution for positive $x$ is $\bar{q}(x)=$ $-q(-x)$. Decomposing into real and imaginary parts and using this property, one obtains

$$
\begin{aligned}
\operatorname{Re} Q\left(\nu, \mu^{2}\right) & =\int_{0}^{1} d x \cos (\nu x)\left(q\left(x, \mu^{2}\right)-\bar{q}\left(x, \mu^{2}\right)\right) \\
& =\int_{0}^{1} d x \cos (\nu x) q_{v}\left(x, \mu^{2}\right), \\
\operatorname{Im} Q\left(\nu, \mu^{2}\right) & =\int_{0}^{1} d x \sin (\nu x)\left(q\left(x, \mu^{2}\right)+\bar{q}\left(x, \mu^{2}\right)\right) \\
& =\int_{0}^{1} d x \sin (\nu x) q_{v 2 s}\left(x, \mu^{2}\right),
\end{aligned}
$$

which relate the valence distribution $q_{v}=q-\bar{q}$ to the real part of the ITDs and the other nonsinglet distribution involving two flavors, $q_{v 2 s} \equiv q_{v}+2 \bar{q}=q+\bar{q}$, to the imaginary part of the ITDs.

All of the equations (16)-(18) involve a known left-hand side (reduced ITDs computed on the lattice and subject to the matching procedure) and integrals of a partonic distribution to be determined. As discussed in detail in Ref. [37], such determination poses an inverse problem, related to the fact that inverse equations are ill defined. Namely, they involve an integral over continuous Ioffe time up to infinity, while on the lattice, one is necessarily restricted to a finite number of determinations of $Q(\nu)$ that cover only a finite range of Ioffe time, from 0 up to 
some $\nu_{\max }$. To reconstruct the distributions, we will follow three ways. The inverse problem stems from having incomplete information and, hence, solving it is not possible without additional assumptions. There is an infinite number of possible assumptions to provide the missing information and, thus, it is important to use as mild ones as possible, in order not to bias the reconstruction procedure. We will perform naive Fourier transforms and we will use two additional ways of handling the inverse problem. First, we will apply the Backus-Gilbert (BG) method [101], originally proposed to be used in PDF reconstruction in Ref. [37]. Second, we will perform reconstruction by fitting the matrix elements using a fitting ansatz for the light-cone PDF, as suggested in Ref. [81].

The BG method minimizes the variance of the solution to the inverse problem; i.e., it maximizes its stability with respect to variation of the data within their errors. This variance minimization condition is a model-independent assumption that provides a unique distribution given a set of input ITDs. For each value of Bjorken- $x$, the minimization condition defines a $d$-dimensional vector $\mathbf{a}_{K}(x)$ (where $d$ is the number of available evaluations of the input ITD), which is an approximate inverse of the kernel function $\mathbf{K}(x)$ [the cosine or the sine function, respectively, for Eqs. (17) and (18)], i.e.,

$$
\Delta\left(x-x^{\prime}\right)=\sum_{\nu} a_{K}(x)_{\nu} K\left(x^{\prime}\right)_{\nu}
$$

where $\mathbf{K}\left(x^{\prime}\right)$ is taken as a $d$-dimensional vector with elements $K\left(x^{\prime}\right)_{\nu}=\cos \left(\nu x^{\prime}\right)$ or $K\left(x^{\prime}\right)_{\nu}=\sin \left(\nu x^{\prime}\right)$. In the ideal case of $d \rightarrow \infty$ evaluations of the ITD spanning the infinite range of Ioffe times, thus defined vector $\mathbf{a}_{K}(x)$ would lead to $\Delta\left(x-x^{\prime}\right)$ being the Dirac delta function $\delta\left(x-x^{\prime}\right)$. In turn, for finite $d, \mathbf{a}_{K}(x)$ leads to an approximation to the Dirac delta function with minimized width. We find the vectors $\mathbf{a}_{K}(x)$ from width minimization conditions, spelled out explicitly e.g., in Ref. [37], which yield

$$
\mathbf{a}_{K}(x)=\frac{\mathbf{M}_{K}^{-1}(x) \mathbf{u}_{K}}{\mathbf{u}_{K}^{T} \mathbf{M}_{K}^{-1}(x) \mathbf{u}_{K}},
$$

where the elements of the $d \times d$-dimensional matrix $\mathbf{M}_{K}(x)$ are given by

$M_{K}(x)_{\nu \nu^{\prime}}=\int_{0}^{1} d x^{\prime}\left(x-x^{\prime}\right)^{2} K\left(x^{\prime}\right)_{\nu} K\left(x^{\prime}\right)_{\nu^{\prime}}+\rho \delta_{\nu \nu^{\prime}}$

and of the $d$-dimensional vector $\mathbf{u}_{K}$ by

$$
u_{K \nu}=\int_{0}^{1} d x^{\prime} K\left(x^{\prime}\right)_{\nu} .
$$

The parameter $\rho$ in $\mathbf{M}_{K}(x)$ regularizes this matrix (Tikhonov regularization [102]; see also Refs. [37,103,104]), making it invertible. The value of $\rho$ should be relatively small in order not to bias the result and not to decrease the resolution of the method. In our study, we find that $\rho=10^{-3}$ is a good compromise, with smaller values introducing large oscillations in the final distributions due to the presence of very small eigenvalues of $\mathbf{M}_{K}(x)$.

Having found the vectors $\mathbf{a}_{K}(x)$ (for both kernel functions), the distributions $q_{v}$ or $q_{v 2 s}$ are reconstructed as

$$
q_{v / v 2 s}\left(x, \mu^{2}\right)=\sum_{\nu} a_{K}(x)_{\nu} \operatorname{Re} / \operatorname{Im} Q\left(\nu, \mu^{2}\right) .
$$

We will also consider a version of the $\mathrm{BG}$ procedure with preconditioning $\left(\mathrm{BG}_{\text {precond }}\right)$, which can be applied in a case where a realistic guess of the solution of the inverse problem is available. In this variant, the kernel function and the desired distribution are rescaled by a function $p(x)$, $\tilde{\mathbf{K}}(x)=\mathbf{K}(x) p(x)$ and $\tilde{q}(x)=q(x) / p(x)$. Then, $\tilde{q}(x)$ embodies the deviation of the reconstructed distribution from $p(x)$. The procedure of calculating the vectors $\mathbf{a}_{K}(x)$ is unchanged, apart from using the rescaled kernel and taking into account the preconditioning function in the final reconstruction equation:

$$
q_{v / v 2 s}\left(x, \mu^{2}\right)=\sum_{\nu} a_{K}(x)_{\nu} p(x) \operatorname{Re} / \operatorname{Im} Q\left(\nu, \mu^{2}\right) .
$$

The other reconstruction technique that we will use is to assume a functional form of a fitting ansatz for the lightcone PDF. This is analogous to procedures used in phenomenological fits of PDFs from experimental data. We will adhere to the simplest reasonable functional form that captures the expected limiting behaviors at low and large $x$ for both $q_{v}$ and $q_{v 2 s}$, in the range $x \in(0,1)$ :

$$
q(x)=N x^{a}(1-x)^{b},
$$

where the exponents $a$ and $b$ are fitting parameters. $N$ is fixed to $1 / B(a+1, b+1)$ for $q_{v}$, where $B(x, y)$ is the Euler beta function, related to the gamma function $B(x, y)=\Gamma(x) \Gamma(y) / \Gamma(x+y)$. This ensures the normalization of the valence distribution to 1 . In the case of $q_{v}+2 \bar{q}, N$ is left as an additional fitting parameter.

The fits are performed minimizing the $\chi^{2}$ function defined as

$$
\chi^{2}=\sum_{\nu=0}^{\nu_{\max }} \frac{Q\left(\nu, \mu^{2}\right)-Q_{f}\left(\nu, \mu^{2}\right)}{\sigma_{Q}^{2}\left(\nu, \mu^{2}\right)},
$$

where $\sigma_{Q}^{2}\left(\nu, \mu^{2}\right)$ is the statistical error of the light-cone ITD $Q\left(\nu, \mu^{2}\right) . Q_{f}\left(\nu, \mu^{2}\right)$ is given by the cosine (sine) Fourier transform of the fitting ansatz (25), respectively, for fits of the real (imaginary) part of ITDs. The fitting function is continuous and, thus, this Fourier transform is not subject to any inverse problem. We will refer to the values of $Q_{f}$ as 
"fitted" ITDs and they are a continuous function of the Ioffe time. Note the fits depend on the maximum Ioffe time $\nu_{\max }$, and we will investigate different choices of this parameter and the sensitivity of the final PDF results to this choice.

\section{LATTICE SETUP}

The underlying matrix elements are the same as the ones used for the computation of quasi-PDFs. Thus, we use the matrix elements of Eq. (1), calculated in Refs. [32,45], corresponding to the Dirac structure $\gamma_{0}$ of the unpolarized PDF case. For quasi-PDFs, the Fourier transform defining the distributions is performed at a fixed nucleon boost $P_{3}$ and the data of Refs. [32,45] concern the cases of $P_{3}=6 \pi / L, 8 \pi / L$, and $10 \pi / L$, corresponding to 0.83 , 1.11 , and $1.38 \mathrm{GeV}$ in physical units, respectively. In the pseudo-PDF approach, the Fourier transform is taken in Ioffe time and hence can profit also from data at low nucleon momenta. Moreover, the double ratio that defines the reduced ITDs requires the knowledge of the zero-boost matrix elements. Thus, for this work, we computed also the cases of $P_{3}=0,2 \pi / L$, and $4 \pi / L$, i.e., $0,0.28$, and $0.55 \mathrm{GeV}$, respectively.

For details of the computational techniques, we refer to the broad description in Ref. [45]. Here, we summarize the main aspects. We use one ensemble of gauge field configurations with two degenerate flavors of maximally twisted mass fermions $[105,106]$ with a clover improvement [107], generated by the Extended Twisted Mass Collaboration (ETMC) [108]. The gauge action is Iwasaki improved [109]. The bare quark mass was tuned to approximately reproduce the physical value of the pion mass $\left[m_{\pi}=130.4(4) \mathrm{MeV}\right]$ and the nucleon mass $\left[m_{N}=932(4) \mathrm{MeV}\right]$ [110]. The lattice spacing is $a=$ $0.0938(2)(3) \mathrm{fm}[110]$ and the lattice volume is $48^{3} \times 96$ sites, which corresponds to a physical lattice extent of $L \approx 4.5 \mathrm{fm}$.

Twisted mass fermions at maximal twist evince automatic $\mathcal{O}(a)$ improvement of physical observables. However, the matrix elements of Eq. (1) are not in this category, apart from the local $(z=0)$ case. Thus, in general, the PDFs calculated in this work have leading cutoff effects linear in the lattice spacing. As shown in Ref. [52], maximal twist can remove some of the $\mathcal{O}(a)$ contributions, but still an explicit specific improvement program is necessary to fully eliminate them. Likewise, an improvement program is needed also for other lattice discretizations, including ones preserving chiral symmetry [52].

In Table I, we summarize the statistics for the computation of matrix elements. While the statistics for $P_{3}<$ $0.83 \mathrm{GeV}$ can be further increased, we keep it lower than the three highest momenta. This is desirable, as we aimed at a similar statistical precision for all data. At these low momenta, the signal-to-noise ratio is very favorable and, thus, similar precision could be achieved already with the
TABLE I. The number of gauge field configurations $\left(N_{\text {confs }}\right)$ and the number of measurements $\left(N_{\text {meas }}\right)$ for each value of the nucleon boost used in this work.

\begin{tabular}{lcrr}
\hline \hline$P_{3}$ & $P_{3}(\mathrm{GeV})$ & $N_{\text {confs }}$ & $N_{\text {meas }}$ \\
\hline 0 & 0 & 20 & 320 \\
$2 \pi / L$ & 0.28 & 19 & 1824 \\
$4 \pi / L$ & 0.55 & 18 & 1728 \\
$6 \pi / L$ & 0.83 & 50 & 4800 \\
$8 \pi / L$ & 1.11 & 425 & 38250 \\
$10 \pi / L$ & 1.38 & 811 & 72990 \\
\hline \hline
\end{tabular}

modest number of measurements reported in Table I. For all momenta, we use a source-sink separation $t_{s}$ of 12 lattice spacings $(1.13 \mathrm{fm})$, which was shown [45] to suppress excited states effects to below the statistical precision of the data. Since the contamination from excited states increases at larger momenta, the additional matrix element computations for this work can be safely assumed to be free from such effects when using $t_{s}=12 a$, at our level of statistical precision.

\section{RESULTS}

\section{A. Bare and reduced matrix elements}

We start by showing bare matrix elements as a function of $z / a$ for the six computed nucleon boosts, from 0 to $1.38 \mathrm{GeV}$; see Fig. 1. We observe that as the hadron

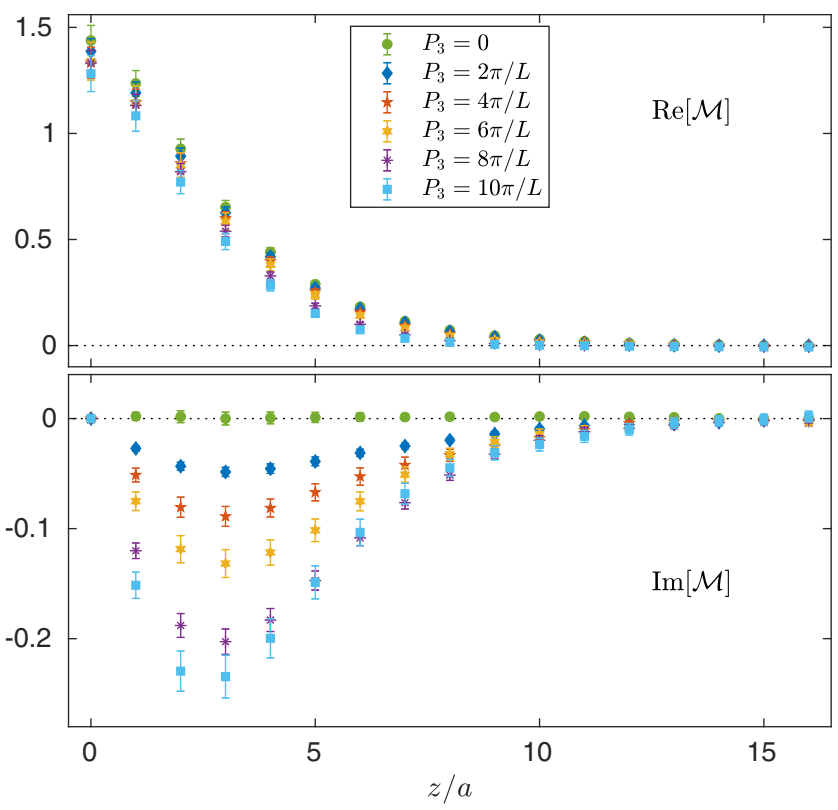

FIG. 1. Real (top) and imaginary (bottom) part of the bare matrix elements $\left[\mathcal{M}\left(\nu, z^{2}\right)\right.$ at fixed $\left.P_{3}\right]$ for the unpolarized PDF. Shown are all nucleon boosts: $P_{3}=0$ (green circles), $P_{3}=2 \pi / L$ (blue rhombuses), $P_{3}=4 \pi / L$ (red five-point stars), $P_{3}=6 \pi / L$ (yellow six-point stars), $P_{3}=8 \pi / L$ (purple asterisks), and $P_{3}=10 \pi / L$ (cyan squares). 


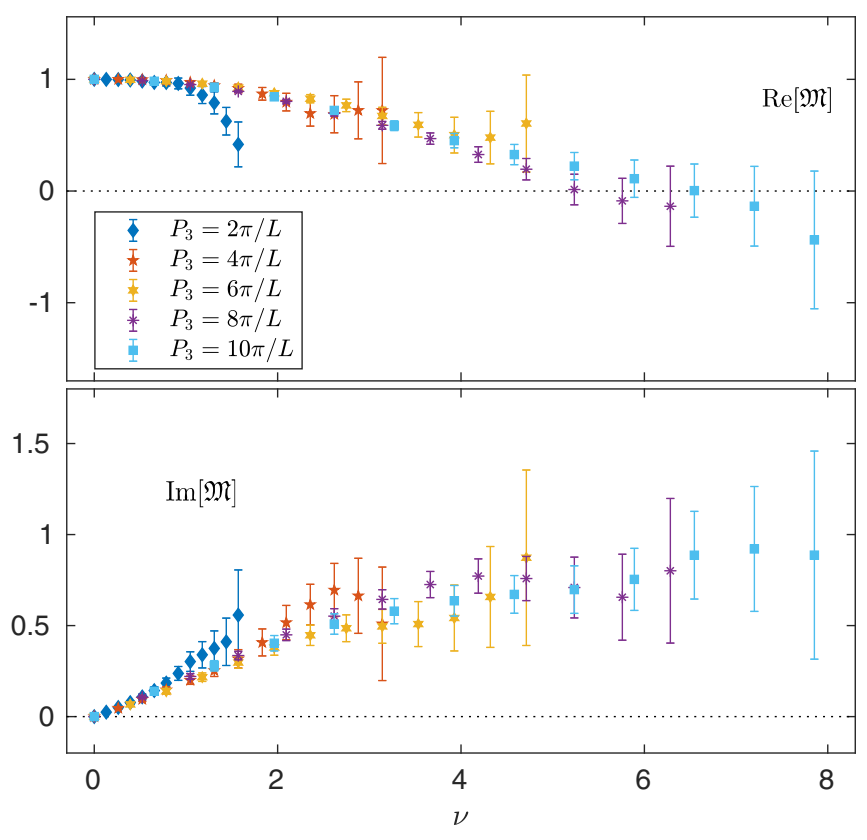

FIG. 2. Real (top) and imaginary (bottom) part of the reduced matrix elements $\left[\mathfrak{M}\left(\nu, z^{2}\right)\right.$ at fixed $\left.P_{3}\right]$ for the unpolarized PDF. Symbols are the same as used in Fig. 1.

momentum is increased, the distribution of the real part of the matrix elements becomes slightly narrower with respect to the length of the Wilson line; i.e., they decay to zero at smaller values of $z$. At the same time, the imaginary part becomes more pronounced for larger momenta. The local matrix element $(z=0)$ is real and contains no divergence. With the employed definition of the vector current, it is only subject to a normalization factor computed in Ref. [111], $Z_{V}=0.7565(4)(19)$, and after multiplication by it, $\mathcal{M}(0,0)$ is compatible with 1 for all nucleon boosts.

We form the reduced matrix elements according to Eq. (2) and plot them against the Ioffe time in Fig. 2, separately for each nucleon boost and for lengths of the Wilson line $z / a \in[0,12]$. We observe that for values of $z / a$ smaller than approximately 8 , reduced ITDs obtained from different combinations of $\left(P_{3}, z\right)$ that lead to the same Ioffe time $P_{3} z$ are compatible with each other within our statistical uncertainties. This suggests that as long as the difference in the scale $1 / z$ is not too large, the scale dependence of reduced ITDs is rather small. The clearest deviations are observed in the real part for the lowest boost, $P_{3}=2 \pi / L$, for $z / a>8$, where the scales $1 / z$ correspond to around $250 \mathrm{MeV}$ and below.

Before we move on to evolution and scheme conversion of the ITDs, we discuss our interpolation procedure at fixed $z^{2}$, which provides the reduced matrix elements at arbitrary, continuous values of the Ioffe time, needed to perform the matching. In Fig. 3, we illustrate our two procedureslinear interpolation between neighboring Ioffe times or a

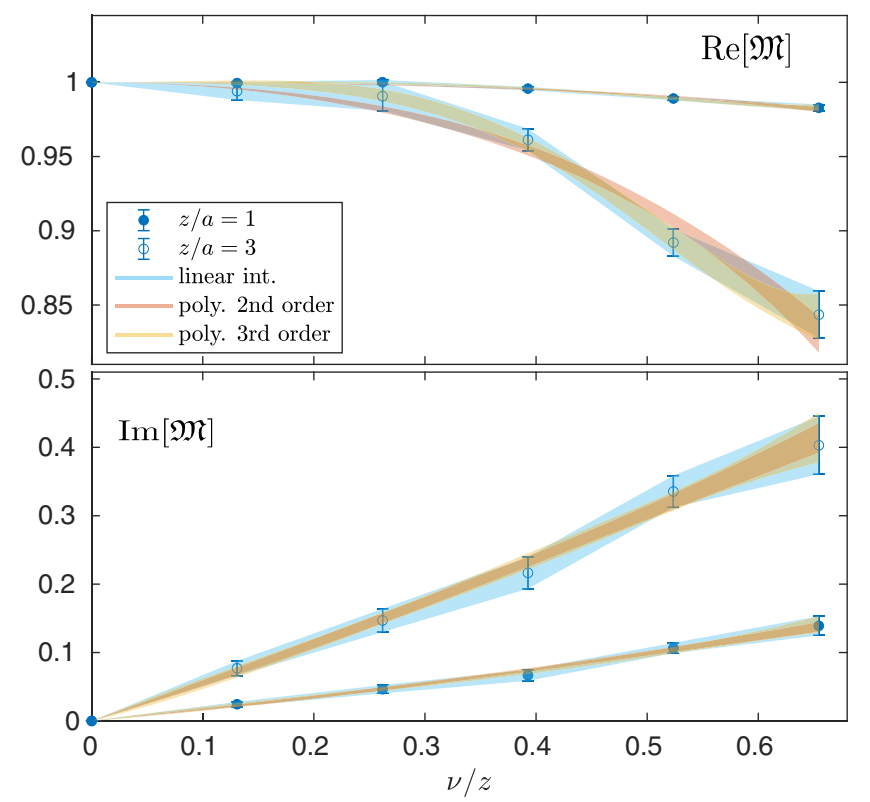

FIG. 3. Illustration of our interpolation procedures to extract the real part (top) and the imaginary part (bottom) of reduced ITDs at fixed $z^{2}$ and arbitrary $\nu$. Shown are the actual lattice data at $(z / a)^{2}=1$ (closed symbols) and $(z / a)^{2}=9$ (open symbols), with linear interpolations between the neighboring points (blue bands) and second (red bands) and third-order (orange bands) polynomial fits to the full Ioffe time dependence. The $\chi^{2} /$ d.o.f. values of the polynomial fits are for the real part: $0.97 / 0.36$ ( $2 \mathrm{nd} /$ 3rd order, $z / a=1)$ and $1.67 / 0.61(z / a=3)$ and for the imaginary part: $0.33 / 0.47(z / a=1)$ and $0.37 / 0.52(z / a=3)$.

polynomial fit to the full Ioffe time dependence (five points corresponding to our five nucleon boosts). For the latter, we find that quadratic or cubic fits are enough to provide good description of the data [in terms of $\chi^{2} /$ d.o.f. that is $\mathcal{O}(1)$ or smaller]. We find an overall consistency between different ways of interpolating, with slight tensions between quadratic and cubic polynomials in certain regions of $\nu$. Below, we also compare these interpolations at the level of the final PDFs, but we take the linear interpolation between neighboring Ioffe times as our preferred method, as it is more conservative and always agrees with both polynomial orders in the fitting method. Note that choosing a polynomial order for this interpolation introduces a mild model assumption and, hence, a difference with respect to other polynomial orders should be considered as a systematic uncertainty. Moreover, it is a priori not clear whether deviations from the fitted curve, observed for some Ioffe times [e.g., for the real part of reduced ITDs at $\nu \approx 0.26$ and $\left.(z / a)^{2}=1\right]$ are mere statistical fluctuations or represent a physical effect and the linear interpolation chooses to keep such deviations and not smoothen them out. However, given the general consistency between all methods, any of them appears to be acceptable at this level of precision. 


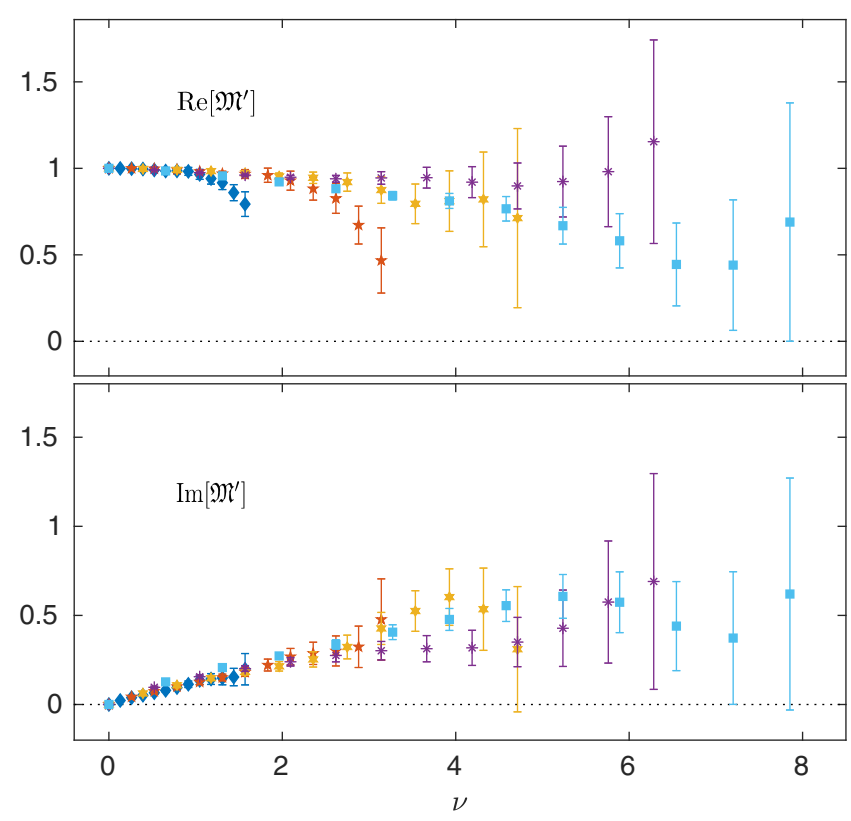

FIG. 4. Real (top) and imaginary (bottom) part of the evolved matrix elements $\left[\mathfrak{M}^{\prime}\left(\nu, z^{2}, \mu^{2}\right)\right.$ at fixed $P_{3}$ ] for the unpolarized PDF. The scale after evolution is $1 / z=\mu e^{\gamma_{E}+1 / 2} / 2 \approx 2.9 \mathrm{GeV}$ and corresponds to the $\overline{\mathrm{MS}}$ scale $\mu=2 \mathrm{GeV}$. Symbols are the same as used in Fig. 1.

\section{B. Evolved and matched ITDs}

The $1 / z$ scale dependence of the ITDs at a fixed Ioffe time is accounted for by the evolution equation (7). We choose to evolve all matrix elements to the scale corresponding to $\mu=2 \mathrm{GeV}$, which will become our final renormalization scale after scheme conversion to the $\overline{\mathrm{MS}}$ scheme. Inspection of the logarithm in Eq. (7) reveals that the scale $\mu=2 \mathrm{GeV}$ corresponds to ITDs being evolved to $z=2 e^{-\gamma_{E}-1 / 2} / \mu \approx 0.72 a$, i.e., around $0.067 \mathrm{fm}$ at our lattice spacing $(1 / z \approx 2.9 \mathrm{GeV})$. For the strong coupling constant, we take the one-loop value at $\mu=2 \mathrm{GeV}$, $\alpha_{s} / \pi \approx 0.129$. Below, we also investigate the dependence on the choice of this value by comparing the results with the ones obtained from $\alpha_{s} / \pi=0.1$, the latter taken in the quenched study of Ref. [80] and close to the value of Ref. [81], $\alpha_{s} / \pi \approx 0.096$, corresponding to a higher-loop coupling used in phenomenology.

The evolved ITDs are slightly closer to a universal curve (see Fig. 4), which is manifested by agreement between ITDs obtained from different combinations of $\left(P_{3}, z\right)$ at the same $\nu$ up to $z / a=10$ in the real part and for all values of $z / a$ in the imaginary part.

The final step of the procedure to arrive at light-cone ITDs is to perform the scheme conversion according to Eq. (8). The outcome is shown in Fig. 5. The agreement between data at a given Ioffe time coming from different momenta and lengths of the Wilson line holds, similarly as for evolved ITDs, up to around 9-10 lattice spacings in the real part and for all values of $z$ in the imaginary part.

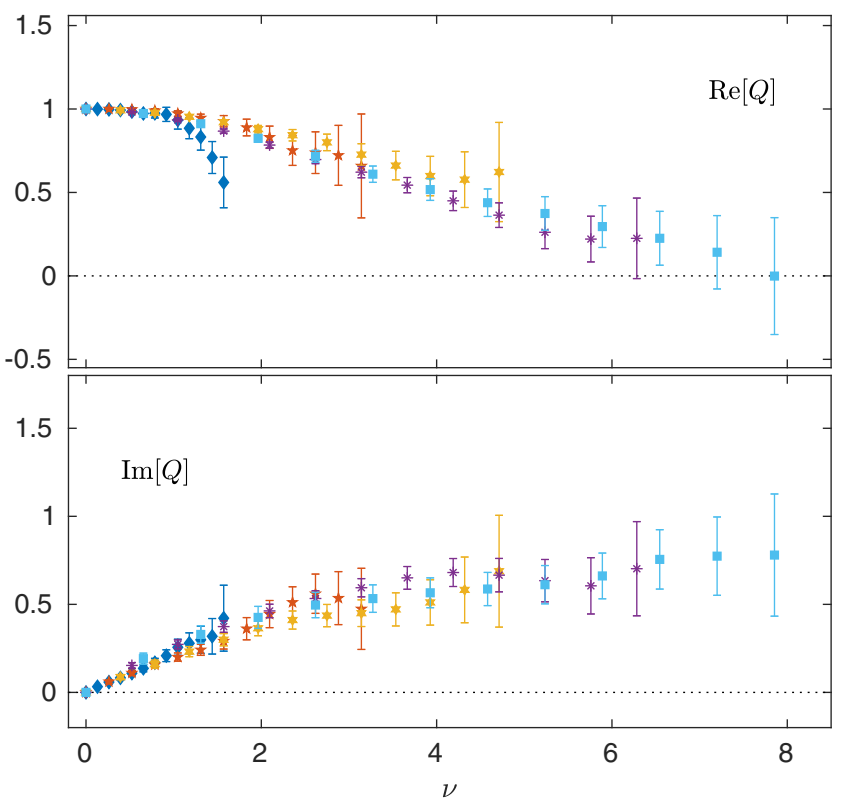

FIG. 5. Real (top) and imaginary (bottom) part of the matched $\overline{\mathrm{MS}}(\mu=2 \mathrm{GeV})$ matrix elements $\left[Q\left(\nu, z^{2}, \mu^{2}\right)\right.$ at fixed $\left.P_{3}\right]$ for the unpolarized PDF. Symbols are the same as used in Fig. 1.

The effects of evolution and scheme conversion are summarized in Fig. 6, where we averaged ITDs corresponding to the same Ioffe time $\nu$, but originating from different combinations of $\left(P_{3}, z\right)$. To avoid contamination from ITDs that are off from a universal curve, we restricted

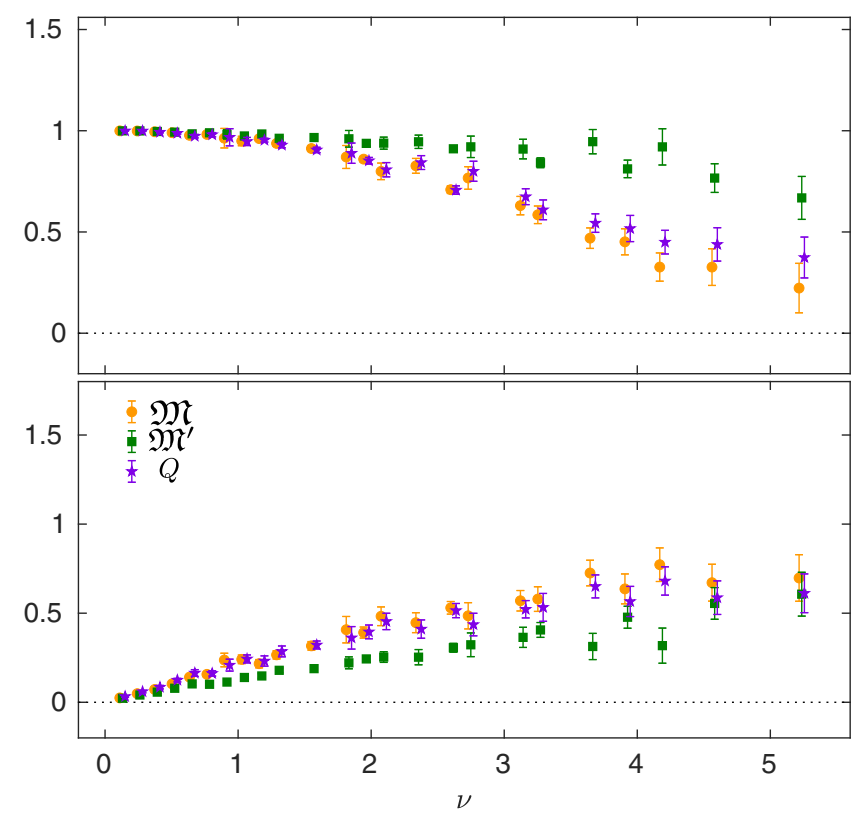

FIG. 6. Real (top) and imaginary (bottom) part of the reduced [ $\mathfrak{M}\left(\nu, z^{2}\right)$; orange circles], evolved [ $\mathfrak{M}^{\prime}\left(\nu, \mu^{2}\right)$; green squares] and matched $\left[Q\left(\nu, \mu^{2}\right)\right.$; purple stars] Ioffe time distributions. The matrix elements corresponding to the same Ioffe time $\nu$ coming from different combinations of $\left(P_{3}, z\right)$ were averaged, keeping only the ones at $z / a \leq 8$. 
the average to cases with $z / a \leq 8$. The effects of evolution and scheme conversion are opposite to each other and, interestingly, approximately equal in magnitude. Thus, the final matched ITDs turn out to be compatible, within our statistical precision, with original reduced matrix elements. It implies that the one-loop matching procedure at the level of ITDs is a small effect, which raises hope that higherorder matching effects are even smaller. Nevertheless, obviously, a two-loop computation is still desired to check explicitly this statement. It is also worth to contrast the oneloop matching for the case of pseudo-PDFs with the one for quasi-PDFs. In the latter case, as e.g., in Ref. [45] that used the same bare matrix elements as the present study, the oneloop matching effects are considerably larger. The matching for quasi-PDFs is performed in $x$ space and the difference between a quasi-PDF and a matched PDF are above $100 \%$ in many regions of $x$. Hence, it is plausible that the matching in the pseudodistribution approach, at the level of ITDs (in $\nu$ space), is more controlled, i.e., less subject to truncation effects.

It is interesting to compare to phenomenological distributions already at the level of ITDs, which can be done by Fourier transforming these distributions back to $\nu$ space. The inverse Fourier transform, an integral of a continuous $x$-dependent distribution, is not subject to an inverse problem and hence, in principle, one can conclude about the agreement of lattice and phenomenology without inverse problem issues involved in reconstructing an $x$-dependent distribution from a finite set of $\nu$-truncated lattice ITDs. In Fig. 7, we compare our matched ITDs

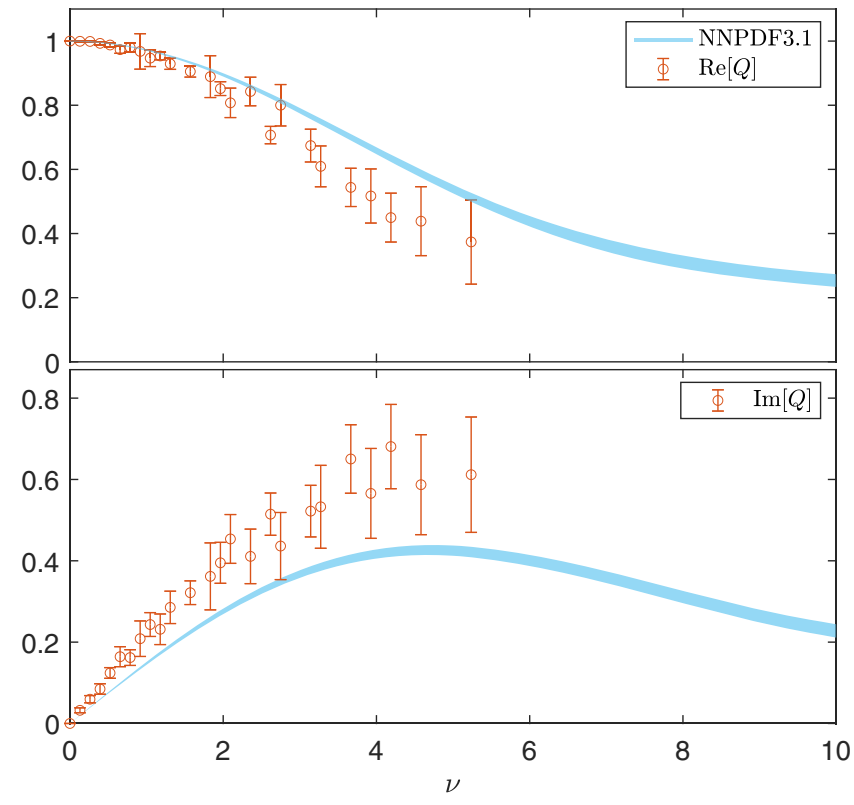

FIG. 7. Real (top) and imaginary (bottom) part of the matched Ioffe time distributions (open circles; $z_{\max } / a=8, \alpha_{s} / \pi \approx 0.129$, $\overline{\mathrm{MS}}$ scheme at $\mu=2 \mathrm{GeV}$ ) compared to NNPDF distributions [112] at the same renormalization scale, inverse-Fourier-transformed to $\nu$ space. $\left(z_{\max } / a=8, \alpha_{s} / \pi \approx 0.129, \overline{\mathrm{MS}}\right.$ scheme at $\left.\mu=2 \mathrm{GeV}\right)$ to inverse-Fourier-transformed NNPDF distributions [112]. The matched ITDs are shown with their statistical errors, as well as with estimated systematic uncertainties, to be defined and discussed below in Sec. IV D. We conclude good agreement of the lattice-extracted ITDs and the phenomenological curves, with a slight tendency of the lattice ITDs to decay a bit faster than NNPDF for the real part and the opposite tendency in the imaginary part. Nevertheless, the agreement is at the level of $1 \sigma-2 \sigma$ for ITDs at most Ioffe times. Overall, this agreement between lattice and phenomenological ITDs is reasonable, giving good prospects for the reconstruction of $x$-dependent distributions. However, there are indications that various systematic effects may be sizable. Moreover, it is clear from the slow decay of NNPDF ITDs that a robust and unambiguous reconstruction of the $x$ dependence may only be achieved when the lattice calculations can get to significantly larger Ioffe times, particularly for distributions involving the imaginary part.

\section{Light-cone PDFs}

We now present results for the unpolarized PDFs obtained from matched ITDs discussed in the previous subsection.

Reconstruction of a PDF from ITDs requires, in principle, the knowledge of the full Ioffe time dependence of the ITDs, from $\nu=0$ to $\nu=\infty$. Obviously, with numerical calculations of ITDs on the lattice, the upper limit $\nu_{\max }$ is necessarily finite. It is desirable to take $\nu_{\max }$ as large as possible, ideally to observe that ITDs have decayed to zero. However, as Fig. 5 suggests, this is difficult with the currently attained nucleon boosts. The real part of matched ITDs approaches zero at $\nu \approx 7-8$, but these Ioffe times are obtained with Wilson line lengths of order $1 \mathrm{fm}$ at the largest boost, which corresponds to very low-energy scales at which the matching procedure is likely to fail. For the imaginary part of ITDs, nonzero values are observed even at $\nu=8$. It is, thus, clear that a robust extraction of the full $x$ dependence requires achieving even larger Ioffe times, especially for the imaginary part that yields the distribution $q_{v 2 s}$. Since the length of the Wilson line is limited by the reliability of the matching procedure and HTE of $\mathcal{O}\left(z^{2} \Lambda_{\mathrm{QCD}}^{2}\right)$, larger Ioffe times need to be reached at larger nucleon boosts. This is, however, difficult for the lattice, which is due to the decaying signal-to-noise ratio with increasing nucleon momentum, as discussed in Ref. [45].

Meanwhile, we are in position to investigate what the currently available range of Ioffe times implies for the $x$ dependence. The key parameter to decide in reconstructing PDFs is the maximum Ioffe time $\nu_{\max }$. Below, we provide reconstructed PDFs for various choices of $\nu_{\max }$, ranging from around 2.6 to 7.9. The former corresponds to taking ITDs obtained from matrix elements with insertions of the operator with Wilson line length up to $z_{\max } / a=4$ lattice 

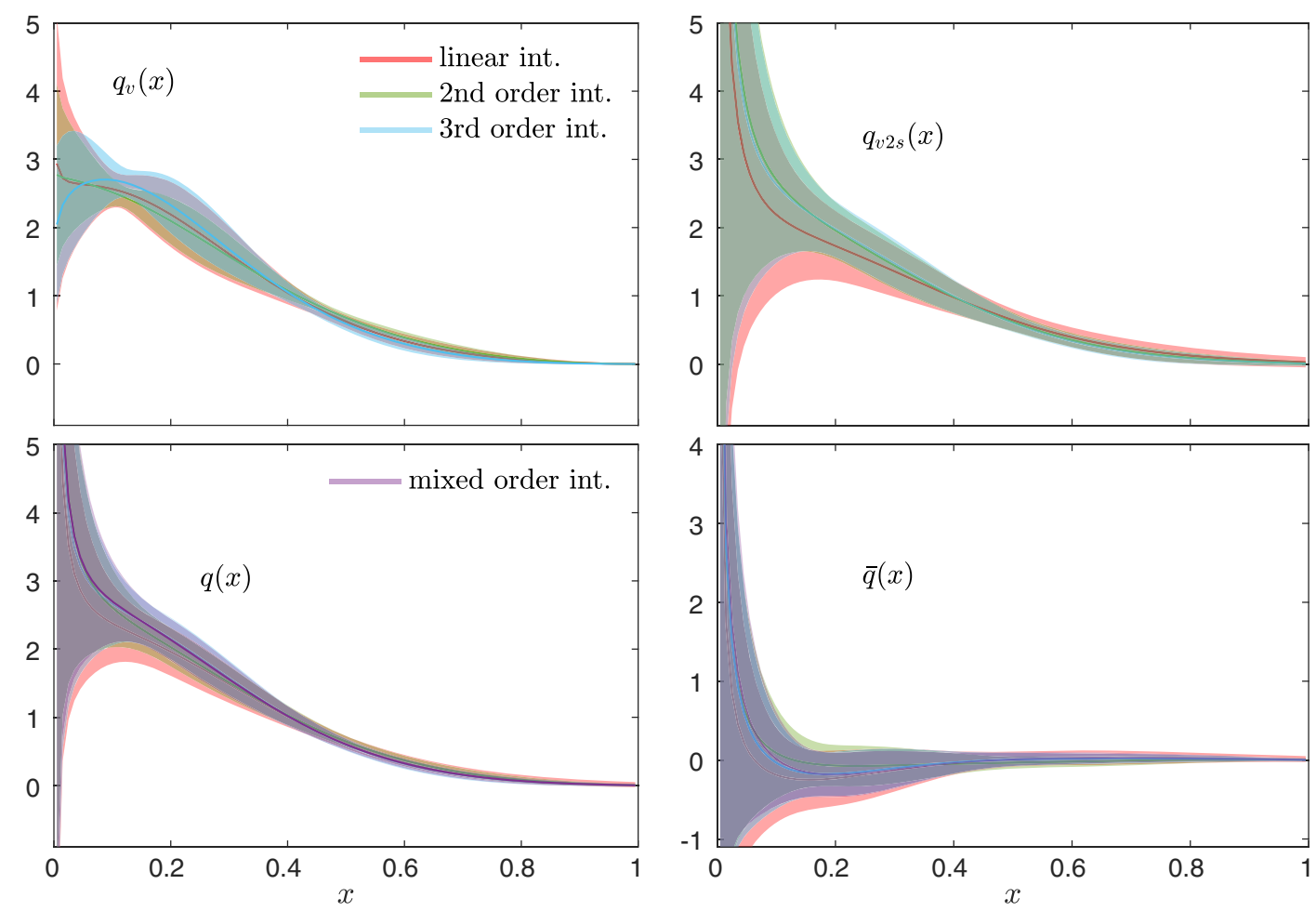

FIG. 8. PDFs obtained with different interpolation methods to access reduced ITDs at continuous Ioffe times and fixed $z^{2}$ : linear interpolation between ITDs at neighboring Ioffe times (red), second (third) order polynomial interpolations from fits to the full Ioffe time dependence [green (blue)] and, where applicable, mixed second (third) order for the imaginary (real) part of ITDs (purple). Light-cone distributions $q_{v}$ (top left), $q_{v 2 s}$ (top right), $q$ (bottom left), and $\bar{q}$ (bottom right). The ITDs are fitted up to $\nu_{\max } \approx 5.2\left(z_{\max } / a=8\right.$ ), $\alpha_{s} / \pi \approx 0.129, \overline{\mathrm{MS}}$ scheme at $\mu=2 \mathrm{GeV}$.

units (around $0.37 \mathrm{fm}$ ) and the latter to 12 lattice units $(1.12 \mathrm{fm})$. It is unclear, a priori, which value of $\nu_{\max }$ ensures reliable matching and good control over HTE. However, given the statistical uncertainties of our data, we adopt a criterion that safe values of $\nu_{\max }$ are those for which the matched ITDs obtained from different combinations $\left(P_{3}, z\right)$ corresponding to the same $\nu$ are consistent with each other. This criterion leads to maximum $z$ of order $8-9$ lattice units (approximately $0.8 \mathrm{fm}$ ), as we have discussed in the context of Fig. 5. In this way, the reached Ioffe times are of order 5-6. At these values of $\nu$, the real part of matched ITDs is already close to 0 , thus giving good hope for the reconstruction of the valence distribution. In turn, the imaginary part of ITDs is still rather far away from zero, which is expected to bring significant uncertainties particularly into $q_{v}+2 \bar{q}$.

Before we investigate the $z_{\max }$ dependence, we compare PDFs at a selected value of $z_{\max }=8 a$ obtained with different interpolation prescriptions to access reduced ITDs at continuous Ioffe times. This is illustrated in Fig. 8, where we show the considered four types of PDFs from linear interpolation between neighboring Ioffe times as well as from polynomial interpolations of second and third order. For the cases of PDFs that mix real and imaginary parts of ITDs $\left(q\right.$ and $q_{s}$ ), we also show a mixed-order interpolation [second (third) order for the imaginary (real) part], motivated by the fact that secondorder polynomials are enough to obtain good fits to the imaginary part at all values of $z^{2}$. As anticipated from comparisons at the level of ITDs, all interpolation methods give consistent PDFs. The approach of the linear interpolation between ITDs at neighboring Ioffe times leads to largest errors and we follow it below as the most conservative choice.

In Figs. 9-11, we show the reconstructed PDFs with $z_{\max } / a=4,8$ and 12 , respectively $\left(\nu_{\max } \approx 2.6,5.2\right.$ and 7.9). The matrix elements used in the reconstruction are shown in the upper row of each figure. It is clear that if $z_{\max } / a=4$ (Fig. 9), the data cover an insufficient range of Ioffe times and, thus, the naive Fourier transform as well as the BG method are simply missing the data and lead to unrealistically looking distributions. However, interestingly, the PDF fitting ansatz approach provides significantly better distributions. The data at small Ioffe times are very precise and guide the fits, leading to precise results at large $x$, in full agreement with the phenomenological curves of NNPDF [112] for all distributions. The fitted matrix elements $Q_{f}\left(\nu, \mu^{2}\right)$ are depicted as bands in the 

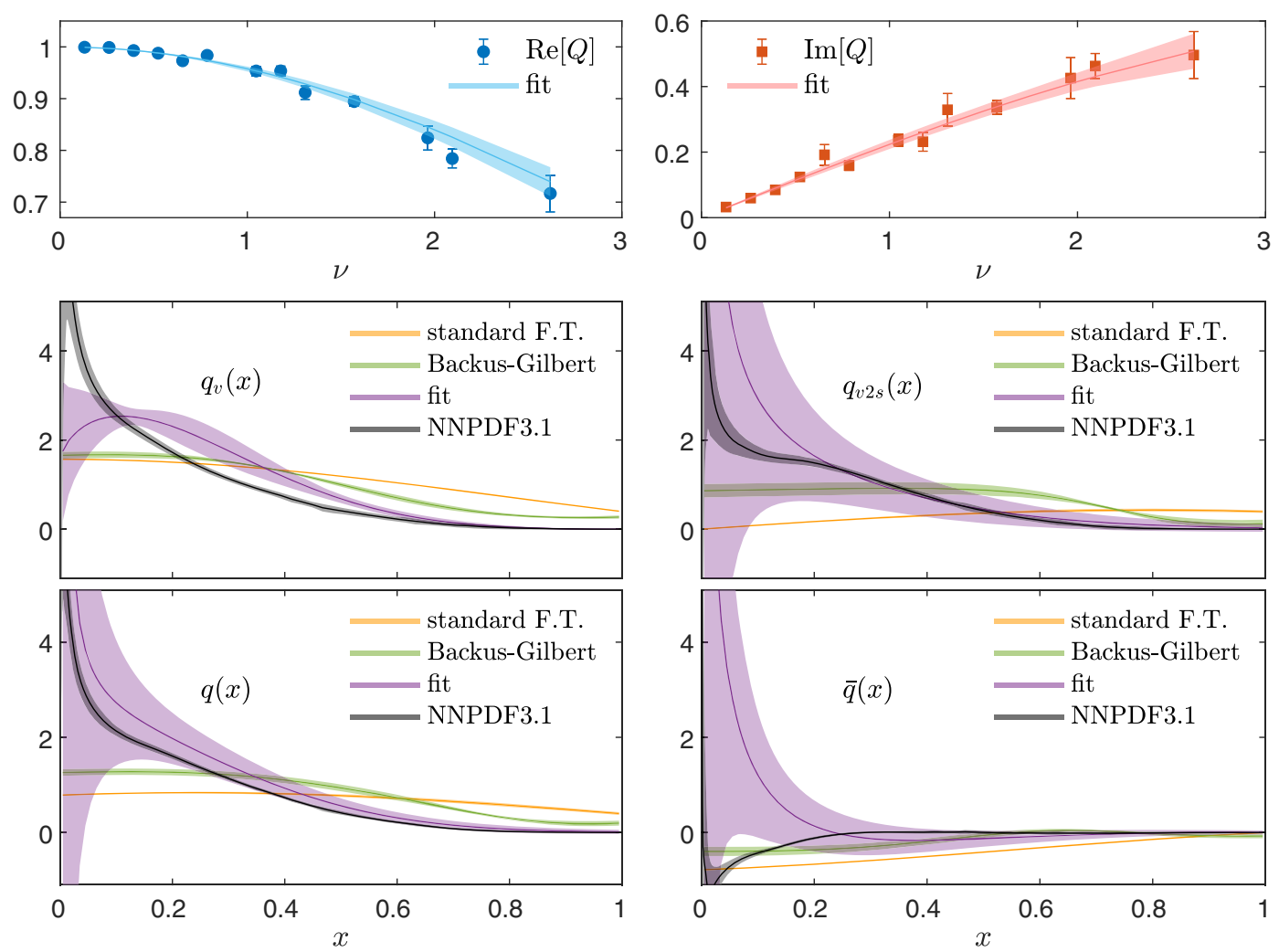

FIG. 9. Top row, left (right) panels: real (imaginary) part of matched ITDs [blue circles (red squares)] and fitted ITDs [blue (red) band]. Middle and bottom row: light-cone distributions $q_{v}$ (middle left), $q_{v 2 s}$ (middle right), $q$ (bottom left), and $\bar{q}$ (bottom right) from three reconstruction methods: naive Fourier transform (orange), Backus-Gilbert (green), and fitting ansatz for the distribution (purple). Shown are also NNPDF phenomenological distributions (gray) [112]. The ITDs are fitted up to $\nu_{\max } \approx 2.6\left(z_{\max } / a=4\right), \alpha_{s} / \pi \approx 0.129, \overline{\mathrm{MS}}$ scheme at $\mu=2 \mathrm{GeV}$.

upper row of Fig. 9 and the fits provide good description of the data $\left[\chi^{2} /\right.$ d.o.f. $\approx 1.0(0.5)$ for the fit of the real (imaginary) part]. The missing data at large $\nu$ manifest themselves in the increasing uncertainty of the reconstructed PDFs at low $x$, in particular for the $q_{v 2 s}$ distribution coming from the imaginary part of matched ITDs. The latter uncertainty propagates itself also to the full distribution $q=q_{v}+\bar{q}$ and to the sea distribution $\bar{q}$, obtained from linear combinations of $q_{v}$ and $q_{v 2 s}$. We note that the full distribution $q$ is consistent with NNPDF for all values of $x$, which, however, holds for $x \lesssim 0.2$ within rather large uncertainties and is partially accidental-the valence distribution is significantly above the curve from phenomenological fits for a wide range of $x$, but this difference is compensated in $q$ by the perfect agreement of $q_{v 2 s}$ in this range.

We want to see now how robust are the PDFs obtained with $\nu_{\max } \approx 2.6$ when increasing the range of Ioffe time used in the fits and whether the other two methods of PDF reconstruction can lead to conclusive distributions. In Fig. 10 , we show the case of $z_{\max } / a=8$, which leads to $\nu_{\max } \approx 5.2$. This range of Ioffe times contains significantly more data points than the range up to $\nu_{\max } \approx 2.6$ and, thus, distributions reconstructed using the naive Fourier transform and, particularly, the BG method start evincing qualitative features of phenomenological PDFs. The additional data at larger $\nu$ entering the fits $\left[\chi^{2} /\right.$ d.o.f. $\approx 0.6(0.4)$ for the fit of the real (imaginary) part] provide more constraints for fitting parameters and their most significant effect is to decrease the PDFs uncertainty at $x \lesssim 0.4-0.5$. This originates from the reduced uncertainty in the fitted matrix elements, i.e., the smaller widths of bands in the upper row of Fig. 10 in the region $\nu \approx 2.6-5.2$, now constrained by the actual lattice data and not simply guided by the low- $\nu$ behavior. However, the bands overlap for the cases of $z_{\max } / a=4$ and $z_{\max } / a=8$ and, thus, the resulting PDFs move only within the uncertainties of the former case. This is encouraging, since it suggests relatively little dependence on $\nu_{\max }$, with the effect of increasing the latter restricted predominantly to giving more precise access to lower $x$ values for the distributions.

Similar conclusions are drawn when further increasing the range of Ioffe times to $\nu_{\max } \approx 7.9\left(z_{\max } / a=12\right)$. For this case, shown in Fig. 11, one needs to keep in mind that the contamination of the large- $z$ ITDs might be significant, as best seen in the deviation of the $z / a \gtrsim 10$ lowestmomentum points from an universal curve (Fig. 5). The extended $\nu$ range has a similar effect on the distributions as 

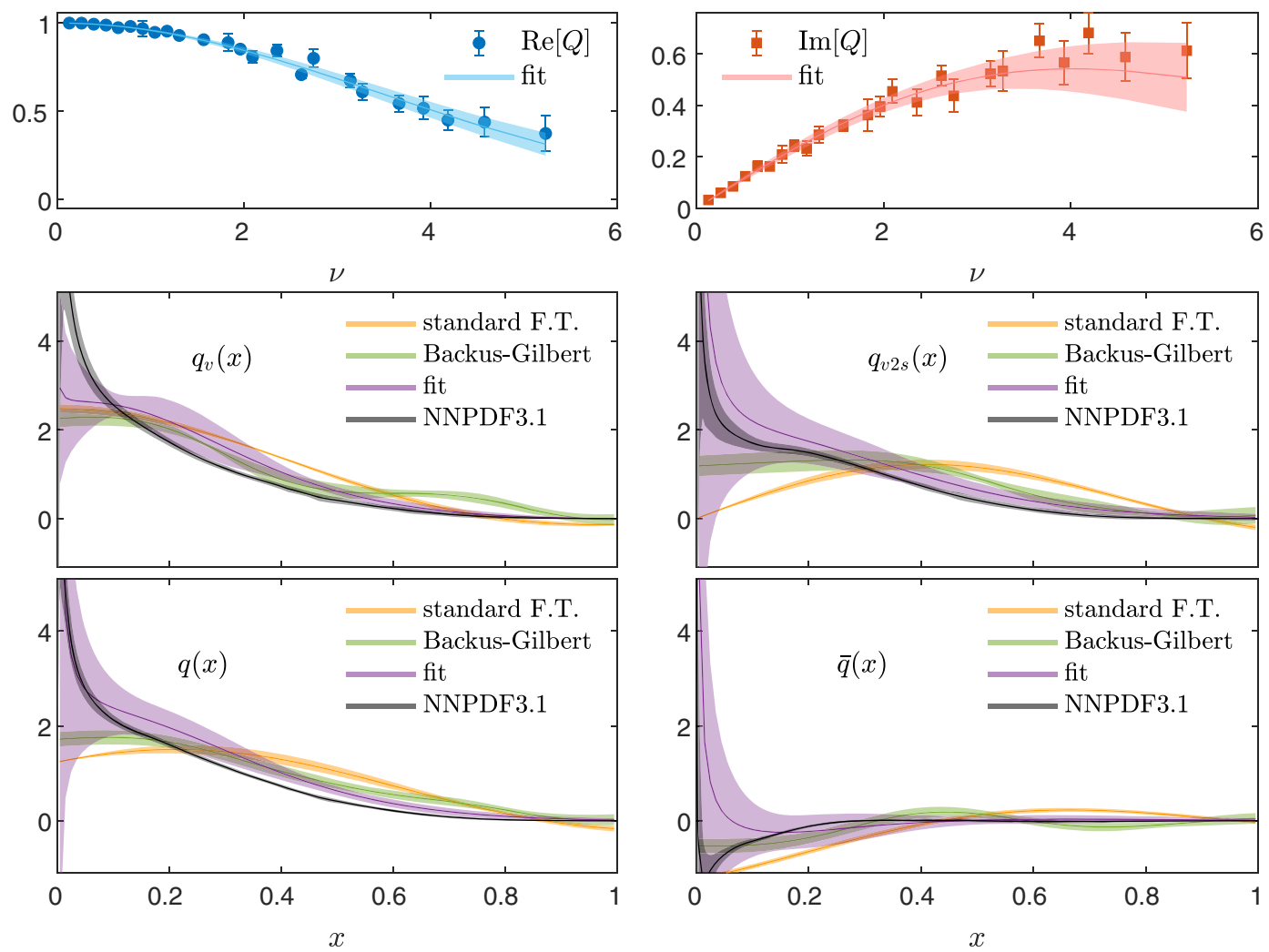

FIG. 10. The same as Fig. 9, but the range of Ioffe times extended to $\nu_{\max } \approx 5.2\left(z_{\max } / a=8\right)$.
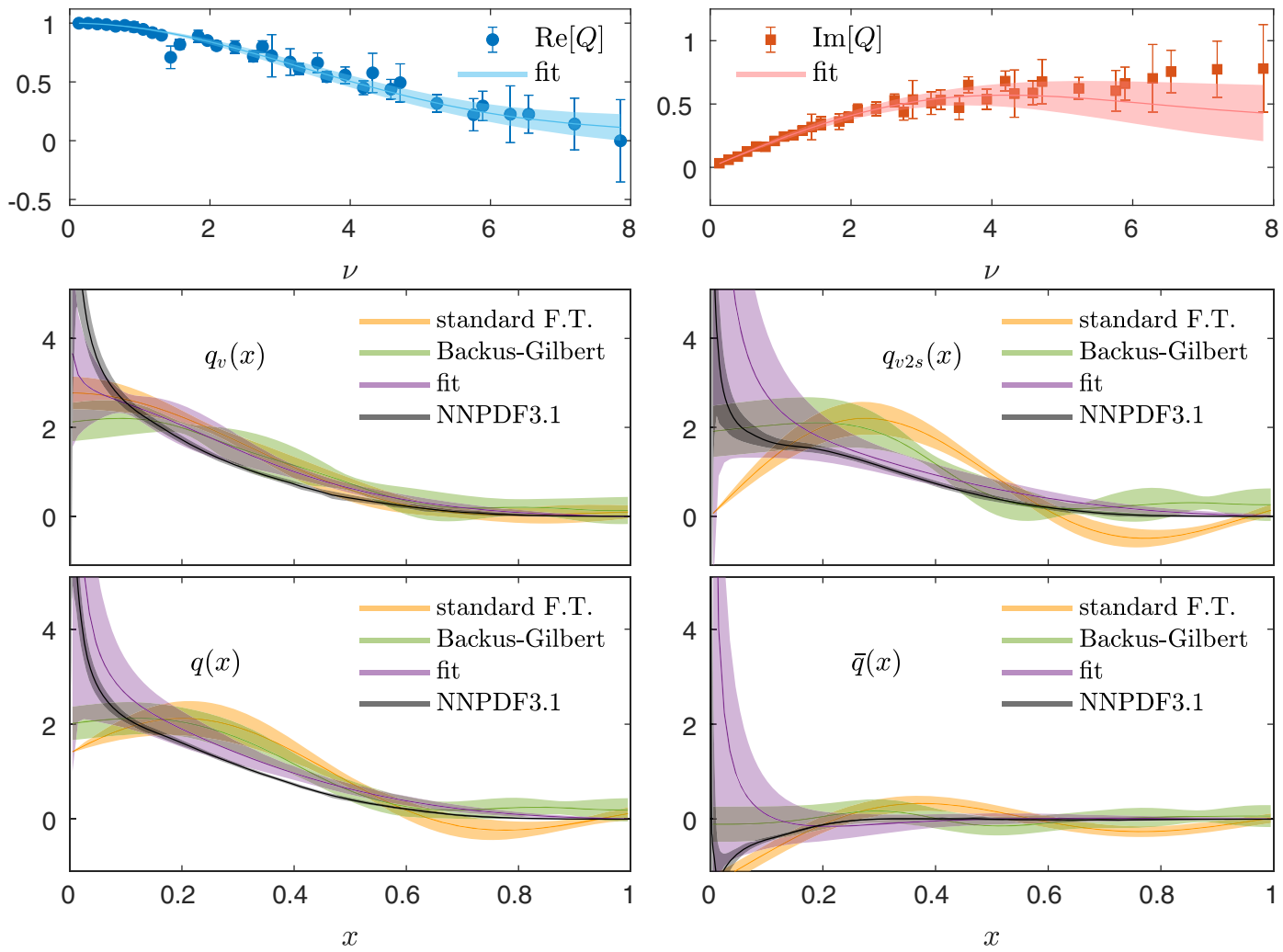

FIG. 11. The same as Fig. 9, but the range of Ioffe times extended to $\nu_{\max } \approx 7.9\left(z_{\max } / a=12\right)$. 
the one when increasing $\nu_{\max }$ from 2.6 to 5.2-PDFs extracted with naive Fourier transform and with the BG method are now qualitatively closer to the phenomenological distributions. The fitting reconstruction again provides good description of the matched ITDs $\left[\chi^{2} /\right.$ d.o.f. $\approx$ 0.5 (0.3) for the real (imaginary) part] and PDFs reconstructed by fitting are only slightly changed with respect to $z_{\max } / a=8$. In fact, they are also compatible with the case of the shortest range of Ioffe time, $z_{\max } / a=4$, within uncertainties. The larger range in Ioffe times again decreases the uncertainty in the low- and intermediate- $x$ regions. For instance, at $x=0.2$, the error in $q_{v 2 s}$ is approximately twice smaller with $\nu_{\max } \approx 7.9$ as compared to $\nu_{\max } \approx 2.6$. An important, if somewhat obvious, effect of enhancing the $\nu$ range is that the inverse problem in the distribution reconstruction becomes less ill defined. This manifests itself in the gradual convergence of all three reconstruction methods. At $z_{\max } / a=4$, the amount of information in the lattice data is scarce and the rather mild additional assumption implicit in the BG method helps only little. The physically motivated assumption provided by the fitting ansatz is, in turn, enough to reconstruct particularly the large- $x$ part of the distributions. When increasing $z_{\max }$, there is more information from the lattice data that shapes the functional form of the distributions and the mild assumption of the BG method is enough to obtain PDFs consistent with the ones from the fitting ansatz. This holds in the full $x$ range, for all considered distributions. The stronger assumption contained in the fitting ansatz is, in turn, verified by adding larger- $\nu$ data, proving that the estimates of large- $x$ PDFs are robust. The access to low $x \lesssim 0.2$ is still limited even with $\nu_{\max } \approx 7.9$ and would require data at yet larger Ioffe times.

The dependence of the results on the Ioffe time range for the three reconstruction methods is summarized in Fig. 12, where we show distributions obtained with $z_{\max } / a=4,6$, $8,10,12$. It clearly demonstrates that the naive Fourier transform is not a plausible method to reconstruct the $x$ dependence. All naively reconstructed distributions are unrobust against changing the $\nu$ range and, at best, certain qualitative agreement is observed with phenomenological PDFs when $z_{\max }$ is large. The BG method does significantly better already with intermediate values of $z_{\max }$. The qualitative features of the PDFs are reproduced, especially at larger $x$. With large $z_{\max }$, there is even quantitative agreement with NNPDF for a wide range of $x$. Comparing $q_{v}$ and $q_{v 2 s}$, the former is more robustly reconstructed due to the faster decay of the real part of matched ITDs in $\nu-$ effectively, there is more "missing information" in the imaginary part. These missing data are also manifested in the irregular behavior of the error, with PDFs at some $x$ having artificially suppressed or enhanced errors. It is rather evident that the most robust way of reconstructing the PDFs is the fitting ansatz approach. The numerically stronger assumption for regulating the inverse problem than the one in the BG method, but physically a well-motivated one, leads to a regular behavior of PDFs with respect to the range of Ioffe times. Distributions obtained from all values of $\nu_{\max }$ are compatible with one another and the parameter $\nu_{\max }$ predominantly controls the uncertainties, in general decreasing the errors at lower values of $x$ when data at larger $\nu$ are included. This is particularly visible for distributions involving the imaginary part of ITDs. For the valence distribution, related to the real part of ITDs by a cosine Fourier transform, the data at large Ioffe times are suppressed - the real part of ITDs decays more quickly in $\nu$ than the imaginary part and, moreover, the cosine function gives more weight to small Ioffe times (apart from very small $x$ ). As a consequence, the valence distribution is very robust against $\nu_{\max }$, with reduction of uncertainties with increasing $\nu_{\max }$ visible only for $x \lesssim 0.1$. In turn, all other distributions receive contributions from the imaginary part of ITDs, weighed by $\sin (x \nu)$. When extending $\nu_{\max }$ from 2.6 to 5.2 and 7.9 , these contributions are numerically potentially more important compared to the case of $q_{v}$, since both the weight is numerically larger for a wide range of $x$ and the imaginary part of ITDs is not suppressed in this regime of Ioffe times. However, we note that after a linear rise of $\operatorname{Im} Q(\nu)$ until $\nu \approx 3$, these ITDs are approximately constant for larger Ioffe times and, thus, their overall contribution is suppressed by the periodicity of the sine weight. Finally, we observe that the sensitivity to $\nu_{\max }$ for distributions related to the imaginary part is only slightly larger than the one of $q_{v}$.

As a further check of systematics, we vary the strong coupling constant in the one-loop evolution and matching of ITDs. In Fig. 13, we compare our choice of this coupling, $\alpha_{s} / \pi \approx 0.129$ at the $\overline{\mathrm{MS}}$ scale of $2 \mathrm{GeV}$, with $\alpha_{s} / \pi=0.1$, equal or close to the value used in other studies [80,81]. Even though the change of $\alpha_{s}$ is rather large, the final distributions are not heavily affected. The largest effect is observed for $x \approx 0.1-0.3$ in $q_{v 2 s}$ and it gets propagated also to the full distribution $q$. However, even this change is well within statistical uncertainties. The robustness of the PDFs with respect to $\alpha_{s}$ is a consequence of the opposite sign of effects in the evolution and the scheme conversion. While the evolved ITDs depend on $\alpha_{s}$ in a more pronounced way, the effect of the scheme conversion brings them back toward reduced matrix elements and, thus, the dependence of the matched ITDs and of the light-cone PDFs on $\alpha_{s}$ is relatively mild. It remains to be established whether this feature holds also at higher-loop orders.

The final question that we want to address in this subsection is how much the preconditioning affects the $\mathrm{BG}$ reconstruction. Above, we observed that the $\mathrm{BG}$ results become increasingly consistent with the ones from the fitting reconstruction when $\nu_{\max }$ is increased. At $\nu_{\max } \approx 7.9$, both methods agree within uncertainties for the full $x$ range of all distributions, as illustrated in Fig. 11. Now, we test 
Naive FT
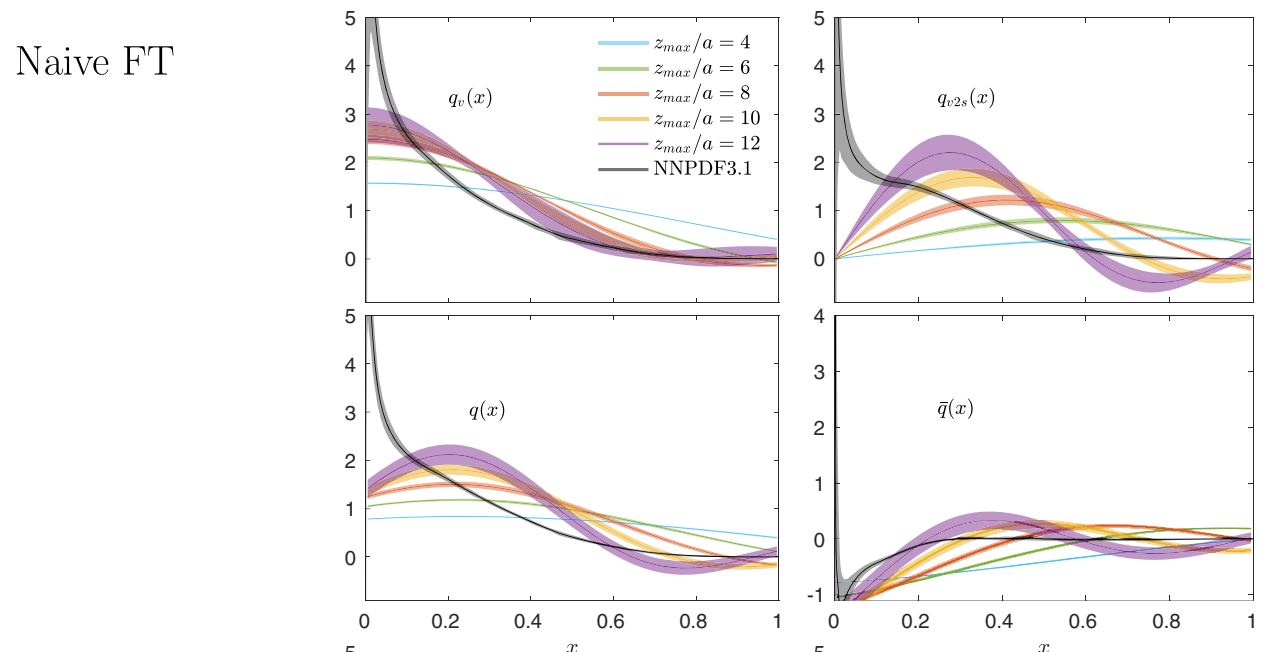

Backus-Gilbert
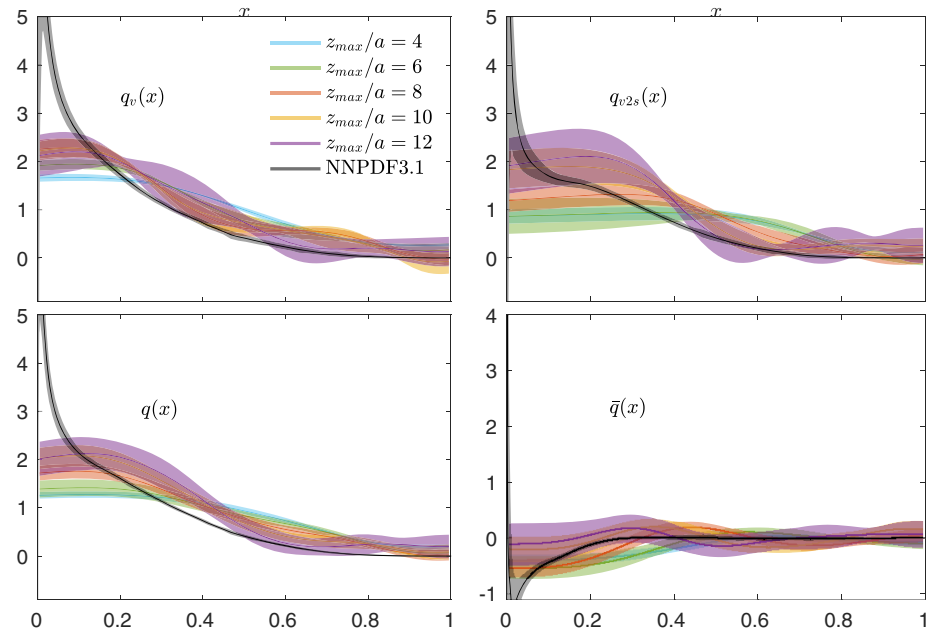

fits
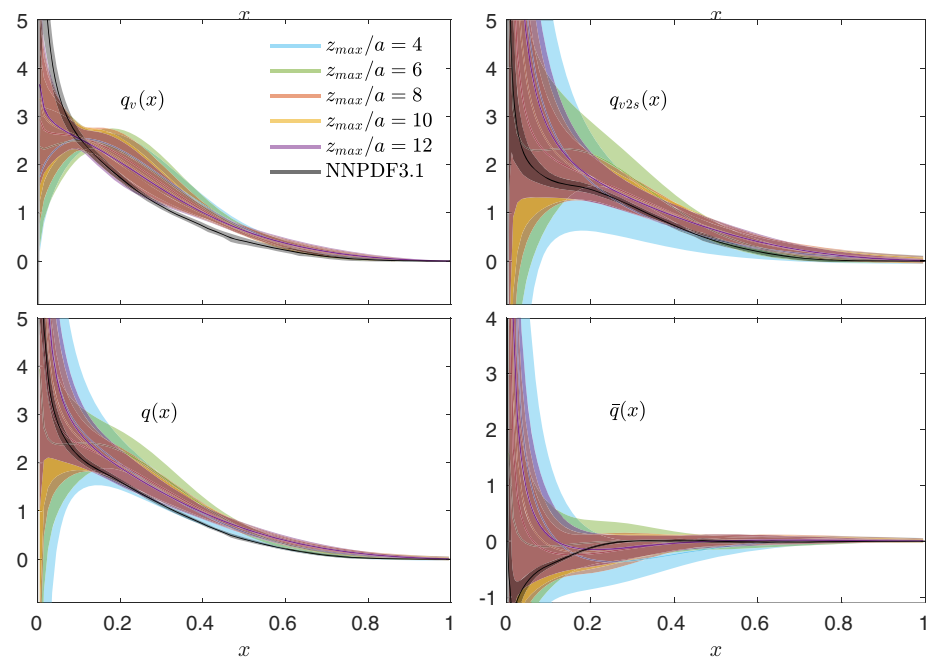

FIG. 12. The dependence of the reconstructed distributions on the range of available Ioffe times, proxied by $z_{\max } / a$. The upper four plots are for $q_{v}, q_{v 2 s}, q$, and $\bar{q}$ with the naive Fourier transform, the middle four for the Backus-Gilbert method and the bottom four for the fitting ansatz reconstruction.

whether this agreement can be extended to lower $\nu_{\max }$ when preconditioning the BG method with the function found in the fitting ansatz approach; i.e., the rescaling function $p(x)$ in Eq. (24) is of the form (25) with parameters $a, b$ (for $q_{v}$ ) or $a, b, N$ (for $q_{v 2 s}$ ) taken to be the central values found in the fits. We emphasize that this does not enforce such form of the distribution but applies the BG criterion of maximal stability of the solution with respect to statistical variance 

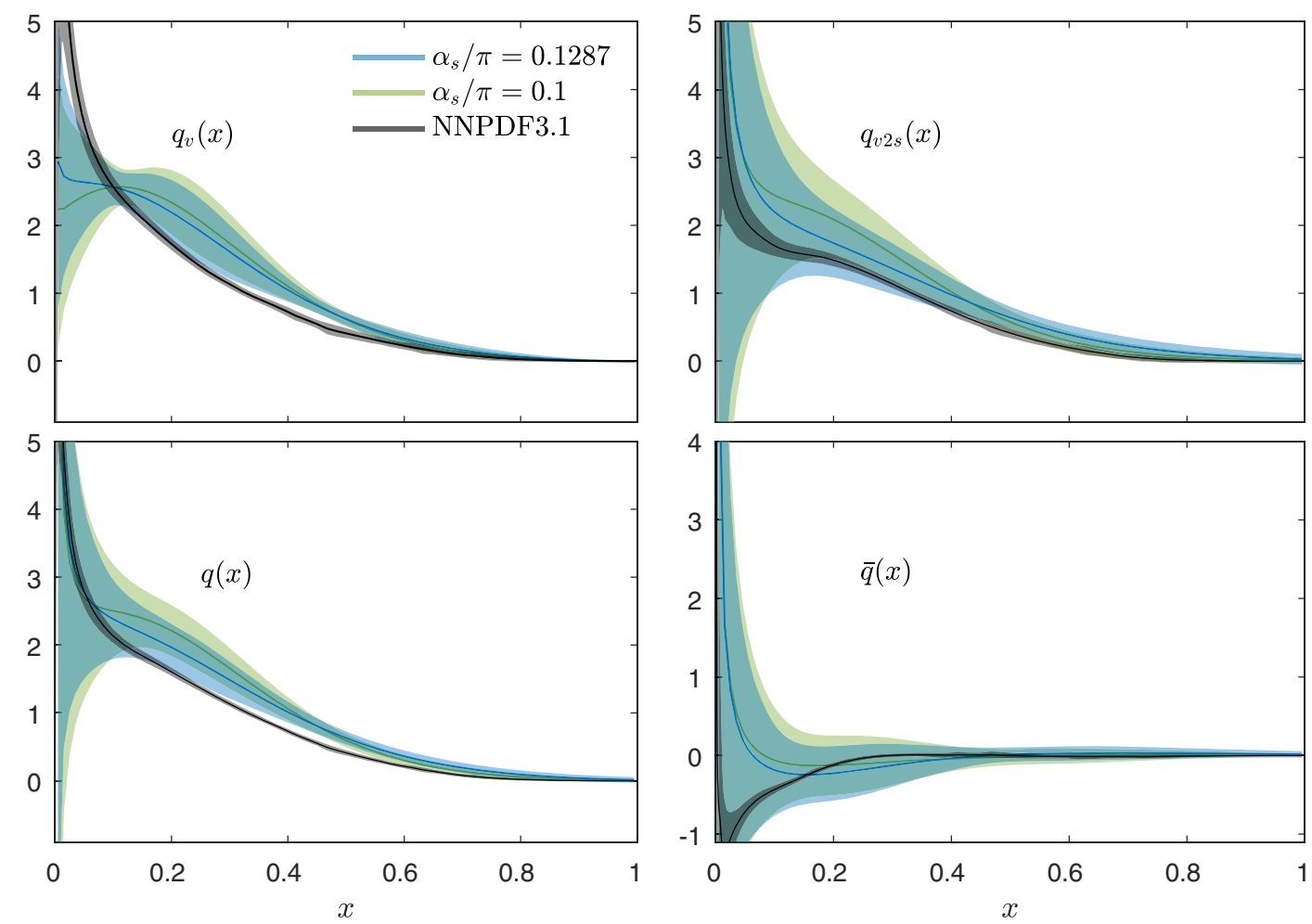

FIG. 13. The dependence of the fitting ansatz reconstructed distributions (upper left, $q_{v}$; upper right, $q_{v 2 s}$; lower left, $q$; lower right, $\bar{q}$ ) on the value of the strong coupling constant $\alpha_{s} / \pi$. The range of Ioffe times in the reconstruction is up to $\nu_{\max } \approx 5.2\left(z_{\max } / a=8\right)$.
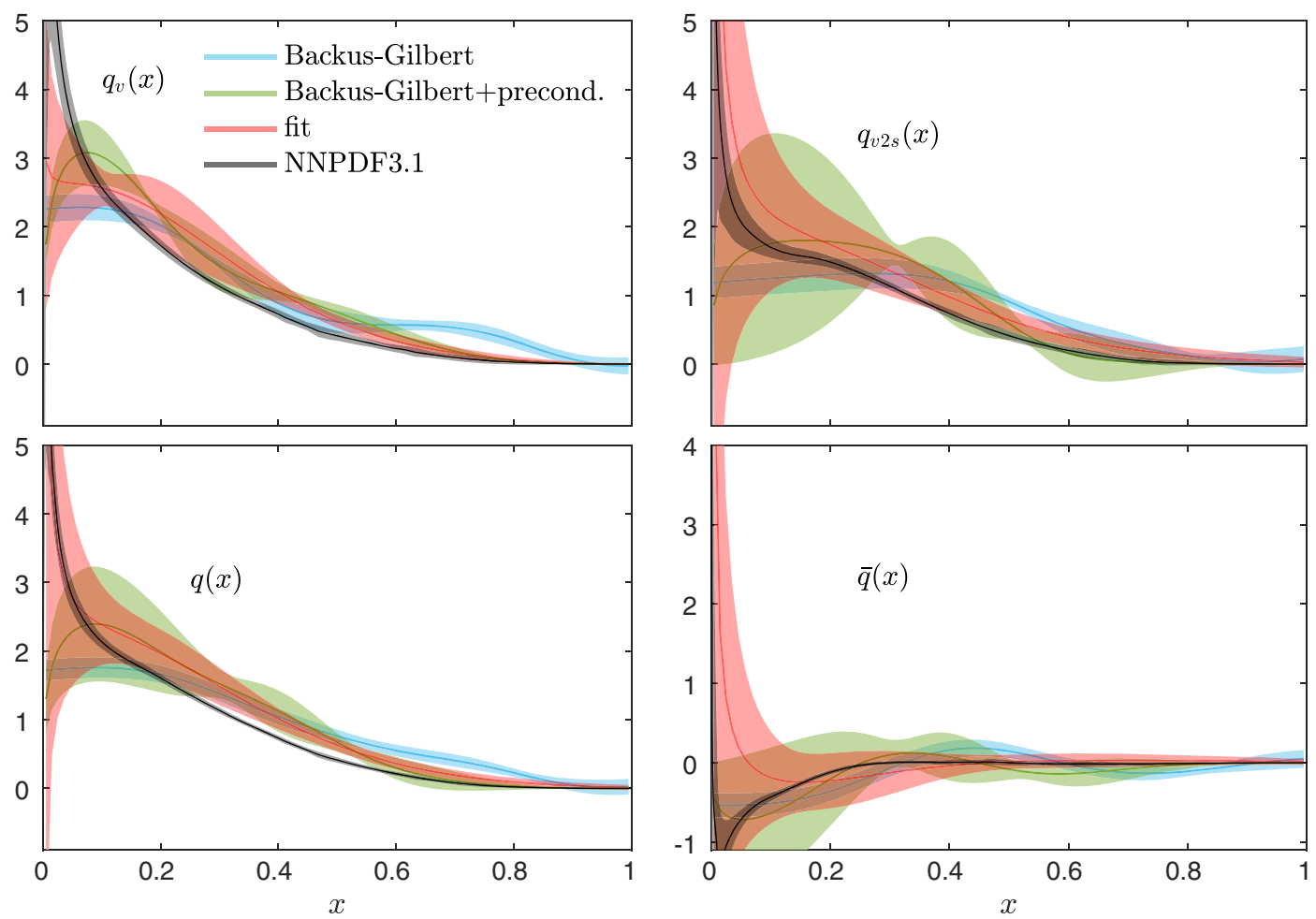

FIG. 14. Comparison of the distributions (upper left, $q_{v}$; upper right, $q_{v 2 s}$; lower left, $q$; lower right, $\bar{q}$ ) reconstructed with the fitting ansatz approach (red) and the Backus-Gilbert method with (green) and without (blue) preconditioning. The range of Ioffe times in the reconstruction is up to $\nu_{\max } \approx 5.2\left(z_{\max } / a=8\right), \alpha_{s} / \pi \approx 0.129$. 
of the data to the deviation of the distribution from the assumed one instead of to the full distribution. In this way, this tests the consistency of the BG assumption regulating the inverse problem with the assumption made in the fitting ansatz approach. In Fig. 14, the comparison of the distributions from the BG method with (green band) and without (blue band) preconditioning to the ones from the fits (red band) is given for $\nu_{\max } \approx 5.2$. We note that preconditioning indeed increases the agreement between BG and fitting results and full consistency is observed between the two at all values of $x$. However, the BG criterion for regulating the inverse problem is not completely equivalent to the fitting ansatz assumption; i.e., the reconstructed function $\tilde{q}(x)=q(x) / p(x)$ [see notation above Eq. (24)] is not equal to 1 . The statistically most prominent effect of preconditioning is observed at large $x$-the preconditioned distributions evince now fully smooth behavior. The agreement of PDFs from $\mathrm{BG}_{\text {precond }}$ with global fits is, however, slightly worse than for the fitting approach, particularly at $x \approx 0.2$ in $q_{v}$. As we noted already for the standard BG method in Fig. 12, one observes irregular behavior of the errors, which are artificially suppressed for some $x$ values and enhanced for others. It can be interpreted that the statistical variance of our ITDs allows only for a very restricted value of the reconstructed PDF at some $x$, while at other values of $x$ significant variation is possible. Naturally, such effects are linked to the missing data in Ioffe time. We note that $\nu \in$ $[0,5.2]$ is the minimum range of Ioffe times needed to observe consistency between $\mathrm{BG}_{\text {precond }}$ and fits. This again points to the fact that $\nu_{\max } \approx 5.2$ is likely to be the optimal value for reconstructing the distributions, i.e., one giving the proper compromise for the Ioffe time range available with our maximal nucleon boost - that should be large to provide enough information but small enough to avoid contamination from HTE and unreliability of the matching procedure applied at too low scales.

\section{Final results with quantified systematic uncertainties}

Having analyzed in detail three distribution reconstruction methods and the dependence of the results on the range of Ioffe times and on the value of the strong coupling constant, we are in position to present our final PDFs. Given the robustness of the fitting ansatz reconstruction with respect to $\nu_{\max }$ and also $\alpha_{s}$, we choose this approach as our preferred one. The central values of our lattice-extracted PDFs use matched ITDs in the range $\nu \in$ $[0,5.2]\left(z_{\max } / a=8\right)$, which fall on a universal curve; i.e., $Q\left(\nu, \mu^{2}\right)$ that can be obtained from different combinations of $\left(P_{3}, z\right)$ are compatible with each other within statistical uncertainties.

Apart from statistical errors of thus defined PDFs, we also add systematic uncertainties. First, we consider the uncertainty related to the range of Ioffe times to be taken in the reconstruction procedure, $\Delta z_{\max }$. For each distribution $q$, we use a conservative definition, that is,

$$
\Delta z_{\max }(x)=\frac{\left|q_{z_{\max } / a=12}(x)-q_{z_{\max } / a=4}(x)\right|}{2} .
$$

Second, we consider the uncertainty from the choice of $\alpha_{s}$, $\Delta \alpha_{s}(x)$ :

$$
\Delta \alpha_{s}(x)=\left|q_{\alpha_{s} / \pi=0.129}(x)-q_{\alpha_{s} / \pi=0.1}(x)\right| .
$$

These two are added to the statistical error and can be considered as the quantified systematics of our result.

In addition, there are further systematic effects in the computation, that cannot be quantified at the present stage. The latter will be subject to extensive follow-up work and will require additional numerical computations and further theoretical developments. An extensive discussion of these effects is given in Ref. [98] and, below, we comment on the most relevant points and follow the strategy of Ref. [49] of assuming plausible magnitudes of the considered effects, as percentages of ITDs values for different Ioffe times. We use estimates corresponding to scenario labeled S2 in Ref. [49], considered to be the most realistic one.

Discretization effects are an obvious source of systematics in lattice calculations. Our results were obtained at a single value of the lattice spacing and, thus, they are potentially contaminated by these effects. To eliminate this uncertainty, simulations at preferably at least two additional lattice spacings are required. Before this is done, in a longer time perspective due to the large cost of such simulations at the physical point, we assume that cutoff effects in our present data can be up to $20 \%$. This number is rather conservative. One argument to support this claim is that larger discretization effects would inevitably lead to the violation of the continuum dispersion relation $E^{2}=P_{3}^{2}+m_{N}^{2}$, where $m_{N}$ is the nucleon mass. Meanwhile, the dispersion relation was tested in Ref. [45] and no deviations from the expected continuum behavior were found. Additionally, related computations of moments of unpolarized PDFs by different groups (see e.g., Ref. [113]) found deviations of $\mathcal{O}(5 \%-15 \%)$ between lattice results at lattice spacings similar to our and the continuum value.

The next source of systematics may be finite volume effects (FVE). Before computations at additional lattice volumes are performed at the physical point to explicitly test them, we rely again on earlier studies, which typically find $\mathcal{O}(1 \%-5 \%)$ effects in related observables, provided that $m_{\pi} L \gtrsim 3$. In our case, $m_{\pi} L \approx 3$. However, as pointed out in Ref. [34] based on calculations in a toy scalar model, FVE in matrix element computations with spatially extended operators may be enhanced and the relevant parameter may be $m_{N}(L-z)$. Since the nucleon mass is much larger than the pion mass, it would effectively not lead to any enhancement of FVE. The worst plausible case 
is if in QCD the parameter controlling FVE becomes $m_{\pi}(L-z)$. However, even then, the values of $z$ that are actually used should not lead to severe FVE. This was confirmed for the $z$-dependent renormalization functions used in the nonperturbative renormalization of quasi-PDFs in Ref. [45]. Overall, to remain conservative, we allow for 5\% FVE in our hypothetical systematic error budget.

Further systematic effects can result from contamination of the signal for the nucleon by excited states with the same quantum numbers. For this uncertainty, investigated in great detail for the matrix elements used in this work, we rely on the conclusion of Ref. [45], where excited state suppression was found within statistical errors of the results. Thus, we take this kind of systematics to be $10 \%$ for all ITDs, i.e., slightly larger than the attained statistical precision.

The perturbative ingredient of our computation is subject to truncation effects. These need to be investigated by calculating at least the two-loop matching. Until this is carried out, the magnitude of this systematic effect is unknown. As we have demonstrated above, changing the $\alpha_{s}$ value for the one-loop formula does not lead to large changes of the PDFs. However, the neglected higher-order effects may still be sizable. The matching to light-cone
ITDs, i.e., the factorization of the pseudo-ITD into its lightcone counterpart and a perturbative coefficient, is also subject to HTE of $\mathcal{O}\left(z^{2} \Lambda_{\mathrm{QCD}}^{2}\right)$. These effects, again, need to be further investigated with dedicated calculations, but they are not expected to be overwhelming with the values of $z$ that are included in the analysis and in view of the rather mild dependence on $z_{\max }$ that we find in this work. Overall, for points discussed in this paragraph, we conservatively attribute $20 \%$ as their potential size.

Our final PDFs are shown in Fig. 15 and compared to global fits of NNPDF [112]. We show three kinds of error bands. The purple one (the most narrow) is exclusively the statistical error of our results. The systematic uncertainties, discussed above, enter in the blue band (quantified systematics from varying the range of Ioffe times and the value of the strong coupling) and in the cyan band (conservatively estimated errors from cutoff effects, FVE, excited state contamination, truncation and HTE). The total uncertainty combines all the separate sources thereof in quadrature.

For all distributions, we find very good agreement with the corresponding phenomenological curve already within statistical errors, while the total error accounts for the remaining small discrepancies in certain regions of $x$ in $q_{v}$ and $q$ (around $x=0.5$ and also $x \lesssim 0.05$ for $q_{v}$ ). This gives
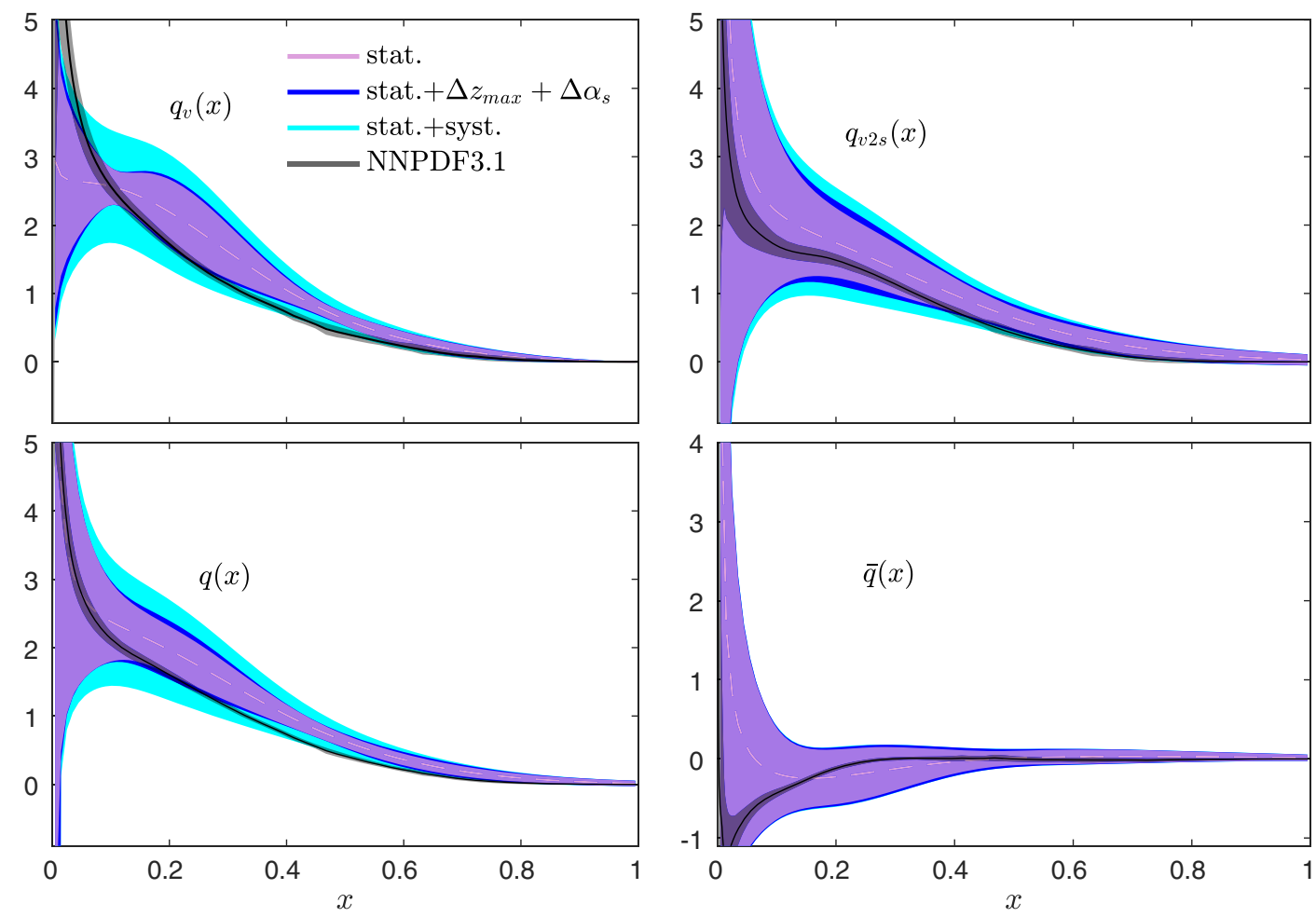

FIG. 15. Final unpolarized PDFs extracted from the lattice (fitting ansatz reconstruction) and compared to global fits of NNPDF [112] (solid black line and dark gray band). Shown distributions: valence $\left(q_{v}\right.$; upper left), valence +2 sea $\left(q_{v 2 s}=q_{v}+2 \bar{q}\right.$; upper right), full $\left(q=q_{v}+\bar{q}\right.$; lower left) and sea $\left(\bar{q}\right.$; lower right). The range of Ioffe times in the reconstruction is up to $\nu_{\max } \approx 5.2\left(z_{\max } / a=8\right)$, $\alpha_{s} / \pi \approx 0.129$. The central value of each PDF is shown with a dashed pink line and the bands represent the statistical uncertainty (purple), the latter with added uncertainty due to the choice of $\nu_{\max }$ and $\alpha_{s}$ (blue) and the total error additionally with estimated uncertainties related to cutoff effects, FVE, excited state contamination, truncation and higher-twist effects (cyan) — see text for more details. 
confidence in the estimates of unquantified systematics, but we emphasize that much work is needed to properly quantify these effects.

The agreement of our final PDFs with NNPDF is striking and shows that a lattice extraction of the full $x$ dependence of PDFs is feasible. It allows us also to draw conclusions about the reliability of such an extraction in different regions of $x$. The most important numerical contributions to PDFs come from regions in Ioffe time where ITDs are large and where the Fourier transform cosine/sine weights are maximal. In practice, this means relatively largest contributions from rather small Ioffe times. For $q_{v}$, only this region has both large ITDs (real part) and a large weight. For $q_{v 2 s}$, the imaginary part of ITDs increases linearly until $\nu \approx 3$ and then stays approximately constant until the largest achieved Ioffe times and the latter fact combined with the periodicity of the sine function numerically suppresses the contributions from this region. Overall, the large- $x$ region is observed to be almost insensitive to the range of Ioffe times when varying $\nu_{\max }$. Thus, it is clear that the large- $x$ part $(x \gtrsim 0.6)$ is reconstructed rather robustly for all distributions. Moreover, one can argue that some other sources of systematics are expected to be small. For instance, since small- $z$ contributions are observed to give largest numerical contributions, $\mathcal{O}\left(z^{2} \Lambda_{\mathrm{QCD}}^{2}\right)$ HTE are probably negligible, there are definitely no enhanced FVE of the type discussed in Ref. [34] and cutoff effects are likely small, since the calculated small- $z$ matrix elements do not differ much from the local $(z=0)$ ones, for which automatic $\mathcal{O}(a)$ improvement holds in our setup. In the intermediate range of $x, x \approx 0.2-0.6$, the uncertainties tend to increase and the one from varying the Ioffe time range becomes non-negligible (as wider range of Ioffe times becomes numerically important; i.e., the cosine/sine weights are varying more slowly, due to their argument being $x \nu$ ), even if it is still subleading. The reconstructed PDFs are less constrained also due to part of the Ioffe time dependence of ITDs missing. The latter becomes especially important in the low- $x$ region $(x \lesssim 0.2)$, where the total error becomes very large, particularly in distributions involving the imaginary part of ITDs that decays more slowly in Ioffe time than the real part.

\section{E. Moment analysis}

In this section, we use the polynomial fits to reduced ITDs at fixed $z^{2}$ to directly extract moments of pseudoPDFs and relate them to moments of light-cone PDFs via the matching equation of Eq. (12). Then, we compare these to moments of our final reconstructed PDFs obtained from a numerical integration of the latter and to phenomenological ones, obtained from numerical integration of the NNPDF distribution [112].

In Fig. 16, we show our lowest two moments from the polynomial fits of second and third order. The fits are performed independently at different discrete $z^{2}$ values but

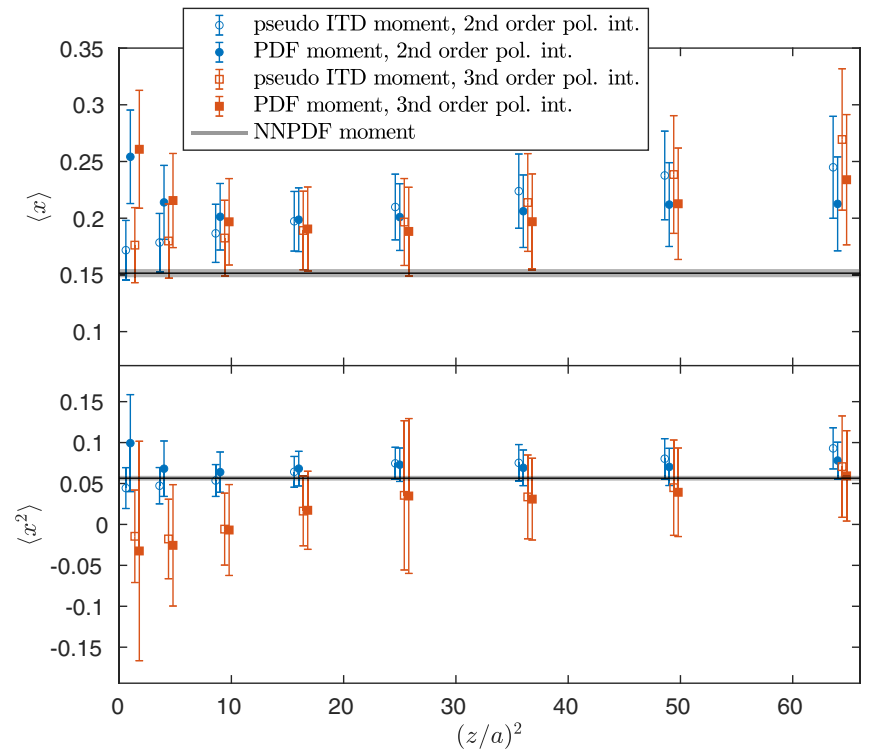

FIG. 16. First (top) and second (bottom) moments of pseudoPDFs (open symbols) and light-cone PDFs ( $\overline{\mathrm{MS}}$ scheme at $\mu=2 \mathrm{GeV}$; closed symbols), coming from second (blue) and third order (red) polynomial fits to the Ioffe time dependence of ITDs at fixed $z^{2}$.

should lead to the same moments up to HTE. The observed independence of moments values within our precision confirms that HTE are indeed comparatively small at least up to $z^{2} \approx 0.56 \mathrm{fm}^{2}$, in line with our conclusions above at the level of final PDFs. The results from the two considered polynomial orders are also consistent with each other; however, the third-order fits are characterized by significantly larger errors. In general, agreement between our extraction and NNPDF moments holds within $1 \sigma$ for most

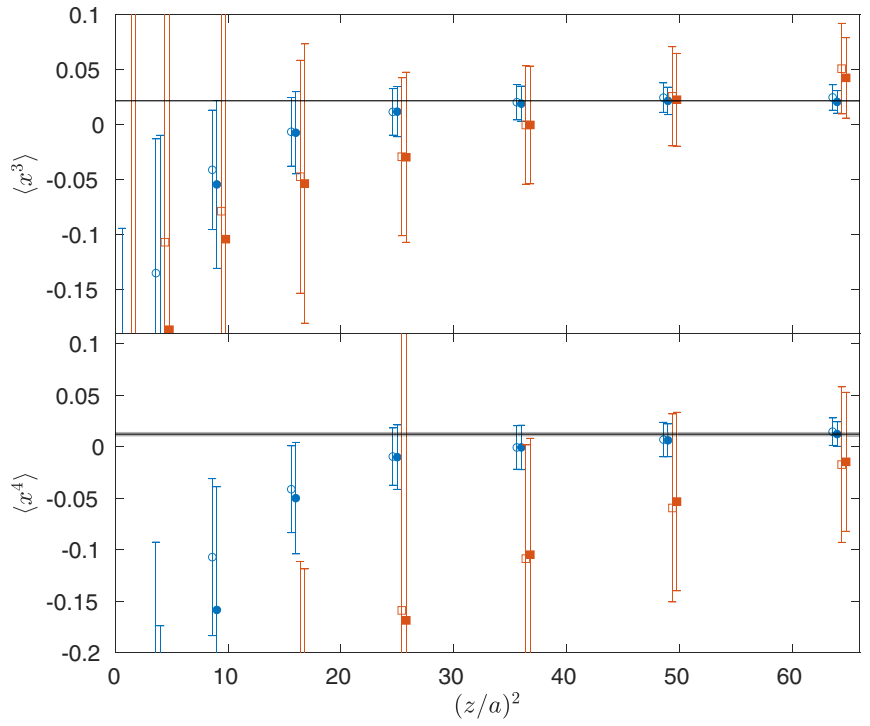

FIG. 17. The same as Fig. 16, but for the third (top) and fourth (moments). 
TABLE II. Comparison of unpolarized PDF moments obtained from numerical integration of the NNPDF distribution [112] with lattice evaluations, from second- and third-order polynomial fits to reduced ITDs at fixed $z^{2} \approx 0.14 \mathrm{fm}^{2}$ and from numerical integration of the final distributions from our three reconstruction approaches [with $\nu_{\max } \approx 5.2\left(z_{\max } / a=8\right), \alpha_{s} / \pi \approx 0.129, \overline{\mathrm{MS}}$ scheme at $\mu=2 \mathrm{GeV}$ ].

\begin{tabular}{|c|c|c|c|c|c|c|}
\hline & \multirow{3}{*}{$\frac{\frac{\text { NNPDF3.1 }}{\text { Integration }}}{\text { Ref. [112] }}$} & \multicolumn{5}{|c|}{ Lattice } \\
\hline & & \multicolumn{2}{|c|}{ Polynomial fits } & \multicolumn{3}{|c|}{ Integration } \\
\hline & & 2nd ord. & 3rd ord. & $\mathrm{nFT}$ & $\mathrm{BG}$ & Fits \\
\hline$\langle x\rangle$ & $0.1515(36)$ & $0.199(29)$ & $0.190(37)$ & $0.23(5)$ & $0.26(6)$ & $0.231(35)$ \\
\hline$\left\langle x^{2}\right\rangle$ & $0.0565(29)$ & $0.068(21)$ & $0.02(5)$ & $0.08(5)$ & $0.12(4)$ & $0.086(29)$ \\
\hline$\left\langle x^{3}\right\rangle$ & $0.0217(6)$ & $-0.01(4)$ & $-0.05(13)$ & $0.04(4)$ & $0.07(4)$ & $0.043(22)$ \\
\hline$\left\langle x^{4}\right\rangle$ & $0.01230(22)$ & $-0.05(5)$ & $-0.40(29)$ & $0.02(4)$ & $0.04(4)$ & $0.023(17)$ \\
\hline
\end{tabular}

cases, with a tendency to slightly too large values for $\langle x\rangle$. Figure 17 illustrates that the third and fourth moments (and obviously all higher ones) cannot be meaningfully extracted at this level of precision. The errors explode, particularly at small $z^{2}$ values, while the more precise second-order polynomial fits at larger $z^{2}$ tend to reproduce the NNPDF values.

In Table II, we compare numbers obtained from the polynomial fits at a selected value of $z^{2} \approx 0.14 \mathrm{fm}^{2}$ and from numerical integration of our final PDFs, from our three reconstruction approaches. Within our uncertainties, all the reported moments are consistent with one another. All NNPDF moments are rather well reproduced, although the uncertainties are relatively large. The value of $\langle x\rangle$ from the integration of the fitting ansatz reconstructed PDFs is around $2 \sigma$ above the phenomenological value, which results predominantly from the systematically too high values of the final PDFs at intermediate momentum fractions. Other reconstruction methods lead to similar values of the moments, but their uncertainties are slightly larger than from the fitting ansatz reconstruction. It is, however, reassuring to observe the agreement of the latter with NNPDF moments, given that they are comparatively more precise with respect to the ones from polynomial fits and statistically meaningful.

\section{SUMMARY}

In this work, we used the pseudodistribution approach to calculate the $x$ dependence of the unpolarized parton distribution functions of the nucleon. The method relies on computations of spatial correlations between boosted nucleon states. The resulting matrix elements are then used to form appropriate ratios that cancel the existing logarithmic and powerlike divergences, defining pseudodistributions in Ioffe time (Ioffe time distributions or ITDs). Pseudo-ITDs are then matched to their light-cone counterparts, objects containing physical information and Fourier conjugate to PDFs. The step of translating the light-cone ITDs to PDFs is highly nontrivial due to the difficulty of obtaining the full Ioffe-time dependence of the former. Thus, it is subject to an inverse problem and advanced reconstruction methods are needed instead of a simple Fourier transform.

We used a robust lattice setup of maximally twisted mass fermions to compute the bare matrix elements at the physical pion mass and with a relatively fine lattice spacing and large lattice volume that should lead to at most modest discretization and finite volume effects. Having computed pseudo-ITDs, we performed the matching procedure to go to the light cone and we reconstructed the $x$ distributions using three methods. As expected, the naive Fourier transform does not lead to robust results. The BackusGilbert methods offers one solution to the inverse problem by assuming that the reconstructed distribution should have minimal variance with respect to the statistical variation of the data. We found that with a large range of the Ioffe-time dependence missing, this criterion is not good enough to reconstruct PDFs. However, with this range being extended, the method gives results convergent with the ones from the third reconstruction approach. The latter assumes a functional form of the light-cone PDFs, as done in phenomenological analyses to extract PDFs from global fits. This assumption, similarly as the one in the BackusGilbert method, regulates the inverse problem and leads, in practice, to well-behaved and robust PDFs. We checked this robustness by investigating the dependence of the reconstructed distributions with respect to the range of included Ioffe times and also the value of the strong coupling constant used in the matching. We found that the large- $x$ region of PDFs is insensitive to this range, while at smaller $x$, ITDs at large Ioffe time lead to variations of the final PDFs only within statistical uncertainties. Obviously, the missing data at these large Ioffe times increase the error of PDF estimates particularly at low $x$. To obtain more precise results there, ITDs need to be computed at larger nucleon boosts. This presents a practical problem for LQCD, since the signal-to-noise ratio in the computation of matrix elements is quickly decaying with each additional unit of momentum. Thus, it is natural that it will be difficult to 
reliably extract the very low- $x$ region. Nevertheless, a large part of the $x$ dependence is possible to determine on the lattice with relatively modest computational resources.

Despite the optimistic results obtained here, it needs to be borne in mind that fully robust lattice-extracted PDFs need to have all relevant sources of systematic effects quantified. Despite the overall excellent agreement with phenomenological distributions, there are intermediate stages where some tension with phenomenology is observed, such as the tendency that $\langle x\rangle$ is too large (at the level of $1 \sigma-2 \sigma$; observed also in the study of Ref. [83]) or the apparently too small (large) values of the real (imaginary) part of matched ITDs at intermediate and large Ioffe times (with similar tension). A reliable quantification of all systematic effects will need a significant load of work in the next few years, involving additional computations on the lattice as well as theoretical developments. Some sources of systematics can be quantified in a straightforward manner by repeating the procedure used in this work for additional ensembles of gauge field configurations, in particular at finer lattice spacings and larger volumes. This will allow us to address, respectively, discretization and finite volume effects. For other kinds of effects, proper strategies of addressing them need to be devised. Truncation effects can only be properly quantified after derivation of higher-loop matching. Higher-twist effects, in turn, can, in principle, be accessed numerically by computing proper matrix elements of more complicated operators, but analytical insight can be invaluable as well.

Thus, the field of extracting $x$-dependent PDFs, as well as other partonic functions, still needs a lot of progress. However, it is clearly reassuring that the quality of our present results is already very satisfactory. Only a few years ago, lattice data for PDFs were limited to only the lowest two or three moments, without realistic perspectives of reconstructing the $x$ dependence. The progress induced by the seminal paper of Ji proposing how to extract the latter and subsequent alternative proposals, in particular the one we used in this work, change the prospects of this field to a huge extent. As demonstrated in this work, the PDFs can indeed be extracted directly from first principles, i.e., from the QCD Lagrangian, and already now this can be done with both qualitative and quantitative agreement with global fits. The latter requires additional work to be fully established and, at this stage, we resorted to plausible hypotheses about the size of some systematic effects.

Given the success of this program for unpolarized PDFs, obvious directions for the future, apart from the discussed analysis of systematics, is to extend the work to polarized PDFs and other kinds of structure functions, in particular generalized parton distributions and transverse-momentumdependent parton distributions, as well as to singlet distributions. All of these directions are challenging but offer an unprecedented opportunity of having crucial insights for the partonic structure of the nucleon, relevant for its deeper understanding both at the theoretical and experimental level.

\section{ACKNOWLEDGMENTS}

K. C. thanks Savvas Zafeiropoulos for many interesting discussions about the pseudodistribution approach at different stages of evolution of this method. M. B., K. C. and A. S. are supported by the National Science Centre (Poland) Grant SONATA BIS No. 2016/22/E/ST2/00013. M. C. acknowledges financial support by the U.S. Department of Energy, Office of Nuclear Physics, Early Career Award under Grant No. DE-SC0020405. This research was supported in part by PLGrid Infrastructure (Prometheus supercomputer at AGH Cyfronet in Cracow). Computations were also partially performed at the Poznan Supercomputing and Networking Center (Eagle supercomputer), the Interdisciplinary Centre for Mathematical and Computational Modelling of the Warsaw University (Okeanos supercomputer) and the Academic Computer Centre in Gdańsk (Tryton supercomputer).
[1] National Academies of Sciences, Engineering, and Medicine, An Assessment of U.S.-Based Electron-Ion Collider Science (The National Academies Press, Washington, DC, 2018), https://doi.org/10.17226/25171.

[2] A. Abdel-Rehim et al., Nucleon and pion structure with lattice QCD simulations at physical value of the pion mass, Phys. Rev. D 92, 114513 (2015); Erratum, Phys. Rev. D 93, 039904 (2016).

[3] M. Oehm, C. Alexandrou, M. Constantinou, K. Jansen, G. Koutsou, B. Kostrzewa, F. Steffens, C. Urbach, and S. Zafeiropoulos, $\langle x\rangle$ and $\left\langle x^{2}\right\rangle$ of the pion PDF from lattice
QCD with $N_{f}=2+1+1$ dynamical quark flavors, Phys. Rev. D 99, 014508 (2019).

[4] C. Alexandrou, S. Bacchio, M. Constantinou, J. Finkenrath, K. Hadjiyiannakou, K. Jansen, G. Koutsou, and A. Vaquero Aviles-Casco, Proton and neutron electromagnetic form factors from lattice QCD, Phys. Rev. D 100, 014509 (2019).

[5] C. Alexandrou et al., Moments of nucleon generalized parton distributions from lattice QCD simulations at physical pion mass, Phys. Rev. D 101, 034519 (2020).

[6] C. Alexandrou, S. Bacchio, M. Constantinou, J. Finkenrath, K. Hadjiyiannakou, K. Jansen, G. Koutsou, and A. Vaquero 
Aviles-Casco, The nucleon axial, tensor and scalar charges and $\sigma$-terms in lattice QCD, Phys. Rev. D 102, 054517 (2020).

[7] C. Alexandrou, S. Bacchio, M. Constantinou, J. Finkenrath, K. Hadjiyiannakou, K. Jansen, and G. Koutsou, Nucleon strange electromagnetic form factors, Phys. Rev. D 101, 031501 (2020).

[8] X. Ji, Parton Physics on a Euclidean Lattice, Phys. Rev. Lett. 110, 262002 (2013).

[9] X. Ji, Parton physics from large-momentum effective field theory, Sci. China Phys. Mech. Astron. 57, 1407 (2014).

[10] X. Xiong, X. Ji, J.-H. Zhang, and Y. Zhao, One-loop matching for parton distributions: Nonsinglet case, Phys. Rev. D 90, 014051 (2014).

[11] H.-W. Lin, J.-W. Chen, S. D. Cohen, and X. Ji, Flavor structure of the nucleon sea from lattice QCD, Phys. Rev. D 91, 054510 (2015).

[12] L. Gamberg, Z.-B. Kang, I. Vitev, and H. Xing, Quasiparton distribution functions: A study in the diquark spectator model, Phys. Lett. B 743, 112 (2015).

[13] C. Alexandrou, K. Cichy, V. Drach, E. Garcia-Ramos, K. Hadjiyiannakou, K. Jansen, F. Steffens, and C. Wiese, Lattice calculation of parton distributions, Phys. Rev. D 92, 014502 (2015).

[14] I. Vitev, L. Gamberg, Z. Kang, and H. Xing, A study of quasi-parton distribution functions in the diquark spectator model, Proc. Sci. QCDEV2015 (2015) 045 [arXiv: 1511.05242].

[15] Y. Jia and X. Xiong, Quasidistribution amplitude of heavy quarkonia, Phys. Rev. D 94, 094005 (2016).

[16] J.-W. Chen, S. D. Cohen, X. Ji, H.-W. Lin, and J.-H. Zhang, Nucleon helicity and transversity parton distributions from lattice QCD, Nucl. Phys. B911, 246 (2016).

[17] J.-W. Chen, X. Ji, and J.-H. Zhang, Improved quasi parton distribution through Wilson line renormalization, Nucl. Phys. B915, 1 (2017).

[18] C. Alexandrou, K. Cichy, M. Constantinou, K. Hadjiyiannakou, K. Jansen, F. Steffens, and C. Wiese, Updated lattice results for parton distributions, Phys. Rev. D 96, 014513 (2017).

[19] A. Bacchetta, M. Radici, B. Pasquini, and X. Xiong, Reconstructing parton densities at large fractional momenta, Phys. Rev. D 95, 014036 (2017).

[20] R. A. Briceño, M. T. Hansen, and C. J. Monahan, Role of the Euclidean signature in lattice calculations of quasidistributions and other nonlocal matrix elements, Phys. Rev. D 96, 014502 (2017).

[21] T. Ishikawa, Y.-Q. Ma, J.-W. Qiu, and S. Yoshida, Renormalizability of quasiparton distribution functions, Phys. Rev. D 96, 094019 (2017).

[22] X. Ji, J.-H. Zhang, and Y. Zhao, Renormalization in Large Momentum Effective Theory of Parton Physics, Phys. Rev. Lett. 120, 112001 (2018).

[23] M. Constantinou and H. Panagopoulos, Perturbative renormalization of quasi-parton distribution functions, Phys. Rev. D 96, 054506 (2017).

[24] C. Alexandrou, K. Cichy, M. Constantinou, K. Hadjiyiannakou, K. Jansen, H. Panagopoulos, and F. Steffens, A complete non-perturbative renormalization prescription for quasi-PDFs, Nucl. Phys. B923, 394 (2017).
[25] X. Ji, J.-H. Zhang, and Y. Zhao, More on large-momentum effective theory approach to parton physics, Nucl. Phys. B924, 366 (2017).

[26] W. Wang, S. Zhao, and R. Zhu, Gluon quasidistribution function at one loop, Eur. Phys. J. C 78, 147 (2018).

[27] J. Green, K. Jansen, and F. Steffens, Nonperturbative Renormalization of Nonlocal Quark Bilinears for Parton Quasidistribution Functions on the Lattice Using an Auxiliary Field, Phys. Rev. Lett. 121, 022004 (2018).

[28] I. W. Stewart and Y. Zhao, Matching the quasiparton distribution in a momentum subtraction scheme, Phys. Rev. D 97, 054512 (2018).

[29] W. Broniowski and E. Ruiz Arriola, Nonperturbative partonic quasidistributions of the pion from chiral quark models, Phys. Lett. B 773, 385 (2017).

[30] W. Broniowski and E. Ruiz Arriola, Partonic quasidistributions of the proton and pion from transverse-momentum distributions, Phys. Rev. D 97, 034031 (2018).

[31] T. Izubuchi, X. Ji, L. Jin, I. W. Stewart, and Y. Zhao, Factorization theorem relating euclidean and light-cone parton distributions, Phys. Rev. D 98, 056004 (2018).

[32] C. Alexandrou, K. Cichy, M. Constantinou, K. Jansen, A. Scapellato, and F. Steffens, Light-Cone Parton Distribution Functions from Lattice QCD, Phys. Rev. Lett. 121, 112001 (2018).

[33] J.-H. Zhang, J.-W. Chen, L. Jin, H.-W. Lin, A. Schäfer, and Y. Zhao, First direct lattice-QCD calculation of the $x$-dependence of the pion parton distribution function, Phys. Rev. D 100, 034505 (2019).

[34] R. A. Briceño, J. V. Guerrero, M. T. Hansen, and C. J. Monahan, Finite-volume effects due to spatially nonlocal operators, Phys. Rev. D 98, 014511 (2018).

[35] G. Spanoudes and H. Panagopoulos, Renormalization of Wilson-line operators in the presence of nonzero quark masses, Phys. Rev. D 98, 014509 (2018).

[36] A. Radyushkin, Structure of parton quasi-distributions and their moments, Phys. Lett. B 788, 380 (2019).

[37] J. Karpie, K. Orginos, and S. Zafeiropoulos, Moments of Ioffe time parton distribution functions from non-local matrix elements, J. High Energy Phys. 11 (2018) 178.

[38] Y.-S. Liu et al. (Lattice Parton Collaboration), Unpolarized isovector quark distribution function from lattice QCD: A systematic analysis of renormalization and matching, Phys. Rev. D 101, 034020 (2020).

[39] H.-W. Lin, J.-W. Chen, X. Ji, L. Jin, R. Li, Y.-S. Liu, Y.-B. Yang, J.-H. Zhang, and Y. Zhao, Proton Isovector Helicity Distribution on the Lattice at Physical Pion Mass, Phys. Rev. Lett. 121, 242003 (2018).

[40] Y. Jia, S. Liang, X. Xiong, and R. Yu, Partonic quasidistributions in two-dimensional QCD, Phys. Rev. D 98, 054011 (2018).

[41] S. Bhattacharya, C. Cocuzza, and A. Metz, Generalized quasi parton distributions in a diquark spectator model, Phys. Lett. B 788, 453 (2019).

[42] C. Alexandrou, K. Cichy, M. Constantinou, K. Jansen, A. Scapellato, and F. Steffens, Transversity parton distribution functions from lattice QCD, Phys. Rev. D 98, 091503 (2018). 
[43] V. M. Braun, A. Vladimirov, and J.-H. Zhang, Power corrections and renormalons in parton quasidistributions, Phys. Rev. D 99, 014013 (2019).

[44] J. Karpie, K. Orginos, A. Rothkopf, and S. Zafeiropoulos, Reconstructing parton distribution functions from Ioffe time data: From Bayesian methods to Neural Networks, J. High Energy Phys. 04 (2019) 057.

[45] C. Alexandrou, K. Cichy, M. Constantinou, K. Hadjiyiannakou, K. Jansen, A. Scapellato, and F. Steffens, Systematic uncertainties in parton distribution functions from lattice QCD simulations at the physical point, Phys. Rev. D 99, 114504 (2019).

[46] S. Bhattacharya, C. Cocuzza, and A. Metz, Exploring twist-2 GPDs through quasidistributions in a diquark spectator model, Phys. Rev. D 102, 054021 (2020).

[47] J.-W. Chen, H.-W. Lin, and J.-H. Zhang, Pion generalized parton distribution from lattice QCD Nucl. Phys. B952, 114940 (2020).

[48] T. Izubuchi, L. Jin, C. Kallidonis, N. Karthik, S. Mukherjee, P. Petreczky, C. Shugert, and S. Syritsyn, Valence parton distribution function of pion from fine lattice, Phys. Rev. D 100, 034516 (2019).

[49] K. Cichy, L. Del Debbio, and T. Giani, Parton distributions from lattice data: The nonsinglet case, J. High Energy Phys. 10 (2019) 137.

[50] H.-D. Son, A. Tandogan, and M. V. Polyakov, Nucleon quasi-parton distributions in the large $N_{c}$ limit, Phys. Lett. B 808, 135665 (2020).

[51] Y. Chai et al., Parton distribution functions of $\Delta^{+}$on the lattice, Phys. Rev. D 102, 014508 (2020).

[52] J. R. Green, K. Jansen, and F. Steffens, Improvement, generalization, and scheme conversion of Wilson-line operators on the lattice in the auxiliary field approach, Phys. Rev. D 101, 074509 (2020).

[53] X. Ji, Fundamental properties of the proton in light-front zero modes, Nucl. Phys. B960, 115181 (2020).

[54] S. Bhattacharya, K. Cichy, M. Constantinou, A. Metz, A. Scapellato, and F. Steffens, Insights on proton structure from lattice QCD: The twist-3 parton distribution function $g_{T}(x)$, Phys. Rev. D 102, 111501 (2020).

[55] V. Braun, K. Chetyrkin, and B. Kniehl, Renormalization of parton quasi-distributions beyond the leading order: Spacelike vs. timelike, J. High Energy Phys. 07 (2020) 161.

[56] S. Bhattacharya, K. Cichy, M. Constantinou, A. Metz, A. Scapellato, and F. Steffens, One-loop matching for the twist-3 parton distribution $g_{T}(x)$, Phys. Rev. D 102, 034005 (2020).

[57] Z. Fan, X. Gao, R. Li, H.-W. Lin, N. Karthik, S. Mukherjee, P. Petreczky, S. Syritsyn, Y.-B. Yang, and R. Zhang, Isovector parton distribution functions of the proton on a superfine lattice, Phys. Rev. D 102, 074504 (2020).

[58] L.-B. Chen, W. Wang, and R. Zhu, Quasi parton distribution functions at NNLO: Flavor non-diagonal quark contributions, Phys. Rev. D 102, 011503 (2020).

[59] Q.-A. Zhang et al. (Lattice Parton Collaboration), Lattice QCD Calculations of Transverse-Momentum-Dependent Soft Function through Large-Momentum Effective Theory, Phys. Rev. Lett. 125, 192001 (2020).
[60] L.-B. Chen, W. Wang, and R. Zhu, Master integrals for two-loop QCD corrections to quark quasi PDFs, J. High Energy Phys. 10 (2020) 079.

[61] S. Bhattacharya, K. Cichy, M. Constantinou, A. Metz, A. Scapellato, and F. Steffens, The role of zero-mode contributions in the matching for the twist-3 PDFs $e(x)$ and $h_{L}(x)$, Phys. Rev. D 102, 114025 (2020).

[62] L.-B. Chen, W. Wang, and R. Zhu, Next-to-next-to-leading order corrections to quark Quasi parton distribution functions, arXiv:2006.14825.

[63] L. Del Debbio, T. Giani, and C. J. Monahan, Notes on lattice observables for parton distributions: Nongauge theories, J. High Energy Phys. 09 (2020) 021.

[64] X. Gao, L. Jin, C. Kallidonis, N. Karthik, S. Mukherjee, P. Petreczky, C. Shugert, S. Syritsyn, and Y. Zhao, Valence parton distribution of the pion from lattice QCD: Approaching the continuum limit, Phys. Rev. D 102, 094513 (2020).

[65] C. Alexandrou, G. Iannelli, K. Jansen, and F. Manigrasso (Extended Twisted Mass Collaboration), Parton distribution functions from lattice QCD using Bayes-GaussFourier transforms, Phys. Rev. D 102, 094508 (2020).

[66] X. Ji, Y. Liu, A. Schäfer, W. Wang, Y.-B. Yang, J.-H. Zhang, and Y. Zhao, A hybrid renormalization scheme for quasi light-front correlations in large-momentum effective theory, Nucl. Phys. B964, 115311 (2021).

[67] C. Alexandrou, K. Cichy, M. Constantinou, K. Hadjiyiannakou, K. Jansen, A. Scapellato, and F. Steffens, Unpolarized and Helicity Generalized Parton Distributions of the Proton within Lattice QCD, Phys. Rev. Lett. 125, 262001 (2020).

[68] J. Bringewatt, N. Sato, W. Melnitchouk, J.-W. Qiu, F. Steffens, and M. Constantinou, Confronting lattice parton distributions with global QCD analysis, Phys. Rev. D 103, 016003 (2021).

[69] W.-Y. Liu and J.-W. Chen, Renormalon effects in quasi parton distributions, arXiv:2010.06623.

[70] C. Alexandrou, K. Cichy, M. Constantinou, J. R. Green, K. Hadjiyiannakou, K. Jansen, F. Manigrasso, A. Scapellato, and F. Steffens, Lattice continuum-limit study of nucleon quasi-PDFs, arXiv:2011.00964.

[71] A. Radyushkin, Nonperturbative Evolution of Parton Quasi-Distributions, Phys. Lett. B 767, 314 (2017).

[72] A. V. Radyushkin, Quasi-parton distribution functions, momentum distributions, and pseudo-parton distribution functions, Phys. Rev. D 96, 034025 (2017).

[73] A. V. Radyushkin, Quark pseudodistributions at short distances, Phys. Lett. B 781, 433 (2018).

[74] A. Radyushkin, Quasi-PDFs and pseudo-PDFs, Proc. Sci. QCDEV2017 (2017) 021 [arXiv:1711.06031].

[75] A. Radyushkin, One-loop evolution of parton pseudodistribution functions on the lattice, Phys. Rev. D 98, 014019 (2018).

[76] A. V. Radyushkin, Generalized parton distributions and pseudodistributions, Phys. Rev. D 100, 116011 (2019).

[77] A. V. Radyushkin, Theory and applications of parton pseudodistributions, Int. J. Mod. Phys. A 35, 2030002 (2020). 
[78] B. L. Ioffe, Space-time picture of photon and neutrino scattering and electroproduction cross-section asymptotics, Phys. Lett. 30B, 123 (1969).

[79] J.-H. Zhang, J.-W. Chen, and C. Monahan, Parton distribution functions from reduced Ioffe-time distributions, Phys. Rev. D 97, 074508 (2018).

[80] K. Orginos, A. Radyushkin, J. Karpie, and S. Zafeiropoulos, Lattice QCD exploration of parton pseudo-distribution functions, Phys. Rev. D 96, 094503 (2017).

[81] B. Joó, J. Karpie, K. Orginos, A. Radyushkin, D. Richards, and S. Zafeiropoulos, Parton distribution functions from Ioffe time pseudo-distributions, J. High Energy Phys. 12 (2019) 081.

[82] B. Joó, J. Karpie, K. Orginos, A. V. Radyushkin, D. G. Richards, R. S. Sufian, and S. Zafeiropoulos, Pion valence structure from Ioffe-time parton pseudodistribution functions, Phys. Rev. D 100, 114512 (2019).

[83] B. Joó, J. Karpie, K. Orginos, A. V. Radyushkin, D. G. Richards, and S. Zafeiropoulos, Parton Distribution Functions from Ioffe Time Pseudodistributions from Lattice Calculations: Approaching the Physical Point, Phys. Rev. Lett. 125, 232003 (2020).

[84] L. Del Debbio, T. Giani, J. Karpie, K. Orginos, A. Radyushkin, and S. Zafeiropoulos, Neural-network analysis of Parton Distribution Functions from Ioffe-time pseudodistributions, arXiv:2010.03996.

[85] U. Aglietti, M. Ciuchini, G. Corbo, E. Franco, G. Martinelli, and L. Silvestrini, Model independent determination of the light cone wave functions for exclusive processes, Phys. Lett. B 441, 371 (1998).

[86] W. Detmold and C. J. D. Lin, Deep-inelastic scattering and the operator product expansion in lattice QCD, Phys. Rev. D 73, 014501 (2006).

[87] V. Braun and D. Mueller, Exclusive processes in position space and the pion distribution amplitude, Eur. Phys. J. C 55, 349 (2008).

[88] Y.-Q. Ma and J.-W. Qiu, Extracting parton distribution functions from lattice QCD calculations, Phys. Rev. D 98, 074021 (2018).

[89] Y.-Q. Ma and J.-W. Qiu, Exploring Partonic Structure of Hadrons Using ab initio Lattice QCD Calculations, Phys. Rev. Lett. 120, 022003 (2018).

[90] K.-F. Liu and S.-J. Dong, Origin of Difference Between Anti-d and Anti-u Partons in the Nucleon, Phys. Rev. Lett. 72, 1790 (1994).

[91] A. J. Chambers, R. Horsley, Y. Nakamura, H. Perlt, P. E. L. Rakow, G. Schierholz, A. Schiller, K. Somfleth, R. D. Young, and J. M. Zanotti, Nucleon Structure Functions from Operator Product Expansion on the Lattice, Phys. Rev. Lett. 118, 242001 (2017).

[92] G. S. Bali, V. M. Braun, B. Gläßle, M. Göckeler, M. Gruber, F. Hutzler, P. Korcyl, A. Schäfer, P. Wein, and J.-H. Zhang, Pion distribution amplitude from Euclidean correlation functions: Exploring universality and highertwist effects, Phys. Rev. D 98, 094507 (2018).

[93] W. Detmold, I. Kanamori, C. D. Lin, S. Mondal, and Y. Zhao, Moments of pion distribution amplitude using operator product expansion on the lattice, Proc. Sci. LATTICE2018 (2018) 106 [arXiv:1810.12194].

[94] R. S. Sufian, J. Karpie, C. Egerer, K. Orginos, J.-W. Qiu, and D. G. Richards, Pion valence quark distribution from matrix element calculated in lattice QCD, Phys. Rev. D 99, 074507 (2019).

[95] J. Liang, T. Draper, K.-F. Liu, A. Rothkopf, and Y.-B. Yang (XQCD Collaboration), Towards the nucleon hadronic tensor from lattice QCD, Phys. Rev. D 101, 114503 (2020).

[96] R. S. Sufian, C. Egerer, J. Karpie, R. G. Edwards, B. Joó, Y.-Q. Ma, K. Orginos, J.-W. Qiu, and D. G. Richards, Pion valence quark distribution from current-current correlation in lattice QCD, Phys. Rev. D 102, 054508 (2020).

[97] K. Can et al., Lattice QCD evaluation of the Compton amplitude employing the Feynman-Hellmann theorem, Phys. Rev. D 102, 114505 (2020).

[98] K. Cichy and M. Constantinou, A guide to light-cone PDFs from Lattice QCD: An overview of approaches, techniques and results, Adv. High Energy Phys. 2019, 3036904 (2019).

[99] M. Constantinou, The x-dependence of hadronic parton distributions: A review on the progress of lattice QCD, in Proceedings of the 38th International Symposium on Lattice Field Theory (2020) [arXiv:2010.02445].

[100] X. Ji, Y.-S. Liu, Y. Liu, J.-H. Zhang, and Y. Zhao, Largemomentum effective theory, arXiv:2004.03543.

[101] G. Backus and F. Gilbert, The resolving power of gross earth data, Geophys. J. Int. 16, 169 (1968).

[102] A. N. Tikhonov, Solution of incorrectly formulated problems and the regularization method, Sov. Math. Dokl. 4, 1035 (1963).

[103] M. V. Ulybyshev, C. Winterowd, and S. Zafeiropoulos, Direct detection of metal-insulator phase transitions using the modified Backus-Gilbert method, EPJ Web Conf. 175, 03008 (2018).

[104] M. Ulybyshev, C. Winterowd, and S. Zafeiropoulos, Collective charge excitations and the metal-insulator transition in the square lattice Hubbard-Coulomb model, Phys. Rev. B 96, 205115 (2017).

[105] R. Frezzotti, P. A. Grassi, S. Sint, and P. Weisz (Alpha Collaboration), Lattice QCD with a chirally twisted mass term, J. High Energy Phys. 08 (2001) 058.

[106] R. Frezzotti and G. C. Rossi, Chirally improving Wilson fermions. 1. O(a) improvement, J. High Energy Phys. 08 (2004) 007.

[107] B. Sheikholeslami and R. Wohlert, Improved continuum limit lattice action for QCD with Wilson fermions, Nucl. Phys. B259, 572 (1985).

[108] A. Abdel-Rehim et al. (ETM Collaboration), First physics results at the physical pion mass from $N_{f}=2$ Wilson twisted mass fermions at maximal twist, Phys. Rev. D 95, 094515 (2017).

[109] Y. Iwasaki, Renormalization group analysis of lattice theories and improved lattice action. II. Four-dimensional non-Abelian SU(N) gauge model, arXiv:1111.7054.

[110] C. Alexandrou and C. Kallidonis, Low-lying baryon masses using $N_{f}=2$ twisted mass clover-improved 
fermions directly at the physical pion mass, Phys. Rev. D 96, 034511 (2017).

[111] C. Alexandrou, M. Constantinou, and H. Panagopoulos (ETM Collaboration), Renormalization functions for $N f=2$ and $N f=4$ twisted mass fermions, Phys. Rev. D 95, 034505 (2017).
[112] R. D. Ball et al. (NNPDF Collaboration), Parton distributions from high-precision collider data, Eur. Phys. J. C 77, 663 (2017).

[113] M. Constantinou, Hadron Structure, Proc. Sci. LATTICE2014 (2015) 001 [arXiv:1411.0078]. 